\section{Cahiers de Narratologie}

Analyse et théorie narratives

$19 \mid 2010$

Images composites, arts pluriels

\title{
La fonction dramatique du dialogue dans les romans médiévaux
}

\section{Corinne Denoyelle}

\section{(2) OpenEdition}

Journals

Édition électronique

URL : http://journals.openedition.org/narratologie/6219

DOI : 10.4000/narratologie.6219

ISSN : 1765-307X

Éditeur

LIRCES

Référence électronique

Corinne Denoyelle, "La fonction dramatique du dialogue dans les romans médiévaux », Cahiers de Narratologie [En ligne], 19 | 2010, mis en ligne le 22 décembre 2010, consulté le 01 mai 2019. URL: http://journals.openedition.org/narratologie/6219; DOI : 10.4000/narratologie.6219

Ce document a été généré automatiquement le 1 mai 2019.

\section{(c) (i) (9)}

Cahiers de Narratologie - Analyse et théorie narratives est mis à disposition selon les termes de la licence Creative Commons Attribution - Pas d'Utilisation Commerciale - Pas de Modification 4.0 International. 


\title{
La fonction dramatique du dialogue dans les romans médiévaux
}

\author{
Corinne Denoyelle
}

Dans un roman, la part dite dialoguée est l'expression de la paresse et de la routine : les personnages parlent pour mettre des blancs dans une page, et par imitation de la vie où il n'y a pas de récit, mais des conversations; il faut donc de temps en temps dans les livres donner la parole aux gens; le contact direct est une économie et un repos pour l'auteur plus encore que pour le lecteur ${ }^{1}$.

1 À quoi servent les dialogues dans un roman ? Auraient-ils, comme le soupçonne sévèrement Blanchot, une simple fonction d'aération, de mise en page et d'effet de réel ? Il n'est plus possible aujourd'hui d'avancer des affirmations si provocantes: la recherche actuelle a montré, au contraire, les difficultés d'écriture que posent les dialogues, les enjeux stylistiques et idéologiques qui s'y rattachent et les subtilités de leurs effets dramatiques. Une telle vision des choses a été remise en question sans que, pour autant, l'articulation entre dialogues et récit soit toujours mise en valeur de façon satisfaisante. Leur rôle a été décrit dans des listes mais non encore analysé dans son fonctionnement narratif.

2 Danielle Coltier, dans un article paru dans Pratiques ${ }^{2}$, classe les paroles de personnages selon leurs diverses fonctions narratives :

- - une fonction d'attestation : « les paroles ne sont reproduites qu'à seule fin d'authentifier les assertions du narrateur, dont elles attestent la justesse »;

- - une fonction de cohésion : les paroles permettent de justifier le passage d'un type de texte à un autre (par exemple du descriptif au narratif) et surtout d'articuler les scènes en signifiant leur clôture, en les annonçant ou en les rappelant : « Parce que [le dialogue] construit, au niveau du discours, une cohérence des actions prétendument représentées, il instaure des relations de conséquence logique entre les scènes racontées et justifie, ainsi, la consécutivité de leur ordre d'apparition. Il est un lieu où se représente la logique de la fiction et où se justifie l'ordre de la narration. »;

- - une fonction dramatique : « les paroles peuvent être des composantes essentielles de l'intrigue, des charnières ouvrant sur des possibles divers, des événements capables 
d'engager des séquences d'actions, par exemple : les bonnes ou mauvaises nouvelles ; la proposition ; l'avertissement ou la menace ; le défi ou l'insulte ; la dénonciation ; la sentence ; le contrat (ou sa rupture), la déclaration d'amour ou de guerre, le serment, la promesse, l'aveu... ";

- - une fonction d'information : le contenu des propos apporte ainsi des informations sur les personnages ou les situations, mais peut aussi apporter un savoir d'ordre paradiégétique (social, philosophique, religieux, idéologique...) ; la forme des propos joue également le rôle d'un indice descripteur des personnages (lexique, manies verbales...) ou de l'ambiance d'une scène.

3 Cette première approche manque de hiérarchisation et d'articulation. Si le rôle du dialogue comme descripteur des personnages a déjà été beaucoup étudié, sa fonction dramatique reste en suspens. Elle consiste en une liste de types de paroles possibles sans que soit montrée la manière dont elles provoquent ou suivent l'action. Plus généralement, que veut-on dire quand on écrit qu'un dialogue « fait avancer l'action »? En quoi crée-t-il un basculement? En quoi sépare-t-il le récit entre un avant et un après? Nous voudrions montrer que cette fonction dramatique consiste en des actes d'engagement et qu'elle recoupe largement la fonction de cohésion en ce qu'elle permet de structurer le récit. La littérature médiévale, qui donne une importance toute particulière à la force du Verbe, sera un lieu pertinent pour analyser cette question.

\section{L'intégration du dialogue à un processus}

Le Moyen Âge fait pleinement sienne cette affirmation d'Austin, Dire, $c^{\prime}$ est faire ${ }^{3}$. L'idée du pouvoir performatif de la parole est largement répandue : un simple mot, une petite phrase peuvent être suivis d'effets spectaculaires. Une question de Perceval aurait permis la guérison du roi Pêcheur et le retour à la prospérité de sa terre ${ }^{4}$; Lancelot entreprend une carrière chevaleresque exceptionnelle pour mériter le qualificatif $d^{\prime} a m i$ que lui a gentiment donné Guenièvre ${ }^{5}$; la mère de Merlin se retrouve enceinte de l'Antéchrist pour avoir parlé à sa sœur sous le coup de la colère $^{6} .$. Aucune parole n'est négligeable et ses effets sont souvent inattendus. Cette importance idéologique de la parole entraîne à tous les niveaux du récit une forte responsabilité du dialogue dans l'organisation de la narration.

5 Nous situant dans la tradition aristotélicienne, nous définirons le récit comme mimèsis praxeos, représentation d'action. Mimèsis est à entendre comme une transposition de l'action dans et par un autre média, le discours. Pour être plus précis, nous distinguerons deux réalités qui concourent l'une et l'autre à la progression dramatique du récit: l'événement et l'action. «Ces notions font l'une et l'autre référence à une modification du cours naturel des choses, en d'autres termes, à une transformation. Mais l'action se caractérise par la présence d'un agent - acteur humain ou anthropomorphe - qui provoque le changement (ou tente de l'empêcher), tandis que l'événement advient sous l'effet de 
causes, sans intervention intentionnelle d'un agent ${ }^{7}$. " Les récits médiévaux comportent plus d'actions que d'événements.

Les séquences narratives qui correspondent aux unités de base du récit, sont fondées sur la décomposition d'une action en ce que Claude Brémond ${ }^{8}$ appelle un processus : « [sa] base est une série élémentaire de trois termes correspondants aux trois temps qui marquent le développement d'un processus : virtualité, passage à l'acte, achèvement. Dans cette triade, le terme postérieur implique l'antérieur : il ne peut y avoir achèvement s'il n'y a eu passage à l'acte, il ne peut y avoir passage à l'acte s'il n'y a eu virtualité. Mais jamais l'antécédent n'implique le conséquent; après chaque fonction, une alternative est ouverte: la virtualité peut évoluer en passage à l'acte ou demeurer virtualité ; le passage à l'acte peut atteindre ou manquer son achèvement. » Le schéma suivant résume ce jeu d'options :

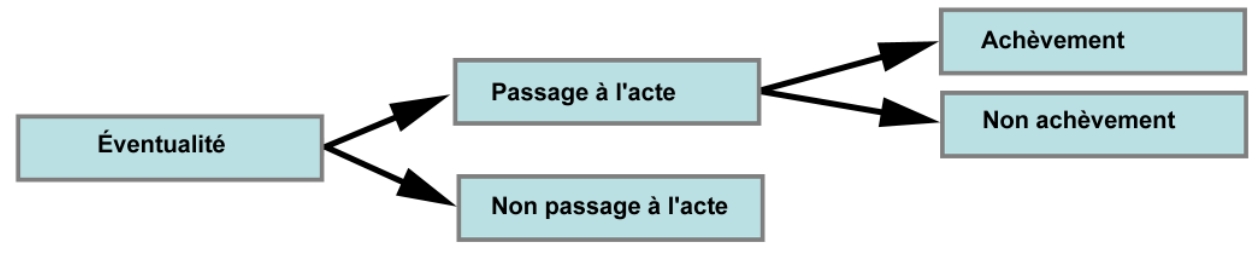

7 Ce schéma correspond à la structure profonde d'une action. Au niveau de l'énoncé, c'est souvent les dialogues qui font passer d'un niveau à un autre. Loin d'être une simple manifestation de l'action ou un simple habillage, ils constituent les charnières qui permettent sa progression. Nous commencerons par observer le fonctionnement des dialogues dans deux scènes romanesques pour en dégager des constantes.

\section{Le dialogue dans une scène typique}

8 Le dialogue joue un rôle structurel dans ce motif récurrent des textes arthuriens qu'est la rencontre entre deux chevaliers sur un pont et le duel qui s'ensuit. Situé à différents niveaux du processus, il en assure l'articulation :

Kahédin et Keu, qui voyagent ensemble, ne peuvent traverser un pont, gardé par le roi Hoël, le propre père de Kahédin, masqué. Keu s'élance pour le combattre mais se trouve très vite abattu.

Quant Kahedins voit et connoist que Kex est abatus, il dist a soi meïsmes que chi n'a sens mestier: a jouster le couvient par force, s'il veut outre passer. Autrement remanroit il decha le pont, s'il ne voloit querre autre passage. Lors descent tout maintenant et regarde a son ceval k'il n'i faille riens k'il i puisse amender ; et quant il s'est apareilliés de la jouste au mieus k'il onques puet, il se met par desus le pont. Atant es vous le roi Hoël venir, ki li crie tant com il puet :

"Dans cevaliers, dans cevaliers, ne vous metés mie sour mon pont, se vous ne volés jouster a moi !

- Sire cevaliers, fait Kahedins, or saciés tout certainnement que mout me soufrisse a cestui point volentiers de cheste jouste, s'il peüst estre autrement! Mais quant je voi apertement k'il me couvient faire folie, je le ferai, u voelle u non."

Lors hurte maintenant cheval des esperons encontre son pere, com chil ki mie nel connoist... (Tristan $1^{9}, 141,8-23$ ) 
9 À ce moment de l'histoire, la joute entre le gardien du pont et le chevalier errant - que tout auditeur de récit arthurien attend - est encore virtuelle, elle ne devient action que sous l'effet du dialogue par lequel le gardien défie son adversaire ${ }^{10}$. Le choix laissé, en apparence, aux personnages s'opère dans et par le dialogue. Certes, celui-ci a été placé là a posteriori pour légitimer la volonté du narrateur de raconter un combat, mais au niveau de la structure du récit, c'est le dialogue qui engage les personnages. Dans ce motif de la joute chevaleresque, les dialogues ne sont pas forcément indispensables pour passer du virtuel à l'acte: l'action peut s'engager sans que son point de bascule soit souligné, c'est le cas par exemple du combat d'Yvain contre Esclador dans le Chevalier au Lion ${ }^{11}$, mais la présence du dialogue permet d'officialiser, de rendre visible ce point de bascule. La forme du défi est le fruit de la tradition arthurienne. Toutefois, elle n'est pas seulement un habillage secondaire : Paul Ricœur a montré la prégnance de la tradition sur la mise en intrigue ${ }^{12}$. Elle contribue à façonner le récit, à donner sa forme à l'agencement des actions. Cette idée est reprise par Denis Bertrand :

De fait, si «éventualité ", " passage à l'acte », " achèvement » décrivent bien les modes d'existence relatifs de l'acte (virtualisation, actualisation, réalisation), leur mise en œuvre dans la narration exige l'intégration des modèles culturels déposés dans la tradition narrative. Eux seuls pourraient, à l'intérieur de ce cadre très ouvert, déterminer des schémas de prévisibilité. Pour passer d'une théorie de l'action à une théorie effective du récit [...], il faut une syntaxe qui détermine un ordre de l'enchaînement infléchi par les configurations culturelles qui le schématisent et rendent l'action racontable ${ }^{13}$.

C'est donc la tradition qui modélise une certaine construction du processus, impliquant que le basculement vers le passage à l'acte se fasse au moyen d'un défi relevé. En effet, le dialogue peut aussi empêcher la réalisation de l'action : dans le Tristan en prose par exemple, les longs débats de quelques chevaliers frondeurs, comme Kahédin et de Dynadan, orientent la joute éventuelle vers le non-passage à l'acte. En refusant le défi qui leur est lancé, ils bloquent la suite du processus.

11 Quelle est la place des dialogues une fois le passage à l'acte effectué ? Dans le duel qui oppose Kahédin à son père, aucun dialogue ne vient interrompre le combat avant que les chevaliers ne s'écartent spontanément l'un de l'autre pour souffler un peu. Les duels sont en effet plutôt «silencieux». Cependant un autre combat de Tristan contre Palamède, durant un tournoi, montre que la présence d'un dialogue est possible :

Quant mesire Tristans voit Palamidés abatu pour ce k'il se voit a tere ausi com il estoit, met il tout maintenant la main a l'espee et court sus a Palamidés, et li donne desus le hiaume un si grant caup com il puet amener d'en haut a la force de ses bras, et puis li dist:

"Palamidés, or tenés ceste : ce est Tristans, vostre amis chiers, qui si grant caus vous set doner!

- Tristan ? ce dist Palamidés. Et ce fu li rois Artus ki les archons vous fist vuidier ! Encore vous en voi je a pié! Certes, je vauroie que chil cevalier ki chi sont laissaissent moi et vous combatre dusc'a outrance. Si m'aït Diex, avant que la nuit venist, cuideroie je grant partie abatre de vostre beubant !" 
Lors li donne desus le hiaume un si grant caup que mesire Tristans s'embronce un poi, et Palamidés dist adonc:

"Tristan, fait il, or tenés ceste ; et se cheste vous atalente, je vous donrai assés des autres. Foi que doi Dieu, de teus dons vous fuisse je larges se nous fuissom seul a seul!

- Palamidés, fait Tristans, or du donner ! Ja certes tant m'en donrés k'il ne vous soient rendu au double! Diex vausist ore que nous fuissom en ceste forest cha devant : au departir verriés vous ki en avroit le pieur part!"

Donc li donne desus le hiaume un si grant caup k'il li fausse le hiaume a fine force, et Palamidés est un poi estourdis, car de mout grant force estoit l'espee descendue.

"Palamidés, fait mesire Tristans, se vous grant caup me donnastes, je n'ai mie a vous failli a cestui point. Bien vous en souviengne! Auques me sui ore aquitiés vers vous."

En tel guise come je vous cont, se combatent li doi chevalier pié a pié en mi l'asamblee, et s'aloient en tel maniere entregabant, non pas de boine volenté, mais du grant doel que cascuns a a son cuer et de la grant rancune que li uns a envers l'autre. Atant es vous vers aus venir le Roi des Chent cevaliers, ki amainne a monsigneur Tristan un cheval:

"Sire, fait il , or tost montés! La nostre gent est tournee a desconfiture, se nous ne lour poom aidier." (Tristan ${ }^{14}$ 2, 176, 11-42)

Les paroles des personnages accompagnent l'action sans en modifier le cours : les chevaliers tout en s'entregabant, se donnent de violents coups qu'ils commentent : "or tenés ceste; et se cheste vous atalente, je vous donrai assés des autres. » Le dialogue vient ici illustrer et rendre plaisant ce combat sans le faire basculer vers son achèvement. D'ailleurs, il n'y a guère d'achèvement: seule l'interruption du Roi des cent Chevaliers vient y mettre fin. Le dialogue est ici de l'ordre du commentaire, il ne fait pas avancer l'action. Tristan y révèle à Palamède son identité de chevalier noir, mais cela ne constitue pas un changement notoire dans l'action dans la mesure où ce dernier s'en doutait déjà.

En revanche, les paroles de personnages peuvent jouer un rôle essentiel dans l'achèvement d'un combat :

(1) Lancelot combat contre Néronneus, un jeune chevalier qui garde un pont. Quand celui-ci demande au héros son identité, il est horrifié de découvrir qu'il combat contre son maître.

Quant Lanselos voit que li cevaliers s'estoit retrais du premier asaut, il se retrait de l'autre part, car bien connoist apertement k'il a grant besoingne de soi reposer. La u li cevaliers s'aloit reposant en tel maniere, esmaiés et espoentés durement, car tant avoit esprouvé Lanselot k'il connissoit certainnement k'il estoit mors s'il ne pooit en aucune maniere trouver merchi en lui, et il s'esmervelloit mout durement ki chiex estoit $u$ il trouvoit si grant pooir et si grant forche.

Quant il s'est une grant pieche reposés et il a bien s'alainne reprise, il conmenche adont a parler et dist :

"Sire cevaliers, nous nous sommes tant combatu ensamble que vous connissiés auques que je sai faire et a vos armes est bien aparant et a m'espee autresi que je voi tainte et vermeille de vostre sanc [...] Et pour ceste cose vauroie je mout volentiers, s'il vous plaisoit, savoir ki vous estes, avant que nous plus feïssom de ceste emprise."

[...] Quant li cevaliers du pont entent que ce est Lanselos du Lac encontre qui il se combat, celui meïsmes ki cevalier le fist, il n'i fait nul autre delaiement, ains jete tout maintenant son escu a tere. Et la $\mathrm{u}$ il voit Lanselot, ki estoit tous en estant et atendoit que li cevaliers se venist combatre a lui, il se laisse, de si haut com il est, caoir a ses piés et dist : 
“Ha ! sire, pour Dieu, merchi ! Pardonnés moi ce que je me sui a vous combatus. [... ]"

Et Lanselos li pardonne volentiers. Lors ostent lor hiaumes, sans autre delaiement faire, et se courent entracoler et conjoiir et se font joie merveilleuse. (Tristan 1, 13, $1-24 ; 14,1-61)$

(2) Après avoir abattu Palamède qui l'avait défié, Tristan attaque le compagnon de celui-ci, Gahériet.

Et Gaheriet, quant il le voit venir vers lui tot apareillié de ferir, il li crie tant com il puet :

"Sire cevaliers, je ne voel la jouste, anchois le vous lais!

- Certes, fait mesire Tristans, dans cevaliers, ensi ne vous poés escaper! Il vous couvient chierement acater l'amour de vostre compaingnon."

Lors hurte ceval des esperons et le fiert si durement k'il abat Gaheriet et le ceval en mi la place, l'un desus et l'autre desous. Et saciés que de celui caoir est Gahéries mout debrisiés. [...] Quant mesire Tristan voit qu'il s'est en tel maniere delivrés de Palamidés et de son compaingnon, il s'en revient a Dynandant et li dist :

"Dynadant, que vous samble de moi ? Vous est il encore avis que je peüsse mon cors desfendre d'un cevalier?

- Sire, ce respont Dynadans, oïl bien ce m'est avis ! Et voirs est, je le connois bien. Encore n'estes vous pas a che menés que je quidoie.

- Hui mais poom nous chevauchier, fait mesire Tristans, car au tournoiement ne retourneroie je pas." [...]

A che s'accordent ambedoi, et il se metent a la voie et laissent Palamidés et Gahériés gisant, ki encore n'avoient pooir d'aus relever tant durement estoient estourdi. (Tristan 2, 186, 34-37 et 187, 1-31)

14 Dans le premier exemple, le basculement de l'acte vers l'achèvement se fait par les paroles du jeune chevalier qui demande à son adversaire de se nommer: "Vauroie je mout volentiers, s'il vous plaisoit, savoir ki vous estes ». Cette demande d'information déclenche une suite d'actes de langage marquant le refus de combattre: engagement à ne plus combattre, demande de pardon, "Pardonnés moi ce que je me sui a vous combatus. », puis pardon accordé. Le processus s'achève par le désengagement de l'un des deux protagonistes. Dans le second exemple, le combat proprement dit se termine sans parole et sans un mot entre les agents qui l'ont mené. En revanche, il est entériné par le dialogue de commentaire entre Tristan et Dynadan : «que vous samble de moi ? ». Ce dialogue permet de prendre acte de la fin du combat sur lequel il porte un jugement ; il signale aussi le désengagement de Tristan du tournoi et son engagement vers une autre action : la reprise de son errance. Le dialogue ne constitue pas le basculement dans l'achèvement, mais il le souligne et constitue le passage à la séquence suivante. Dans le premier cas, le dialogue provoque la fin de l'action, dans le deuxième, il la commente.

Nous dirons donc que, dans le déroulement d'un processus, les paroles de personnage peuvent avoir deux fonctions: soit elles engagent les personnages (ou les désengagent) et font basculer le processus de l'éventuel à l'acte ou de l'acte à son achèvement; soit elles ne font qu'illustrer l'acte ou son achèvement. 


\section{Le dialogue dans une scène non typique}

16 Nous chercherons à vérifier cette première conclusion par l'étude d'une scène non typique. La première épreuve d'Éracle ${ }^{15}$ fournira un exemple d'action: le jeune esclave, qui a reçu de Dieu un pouvoir de discernement, rapporte à l'empereur la pierre magique qu'il vient d'acheter et s'engage à en vérifier la valeur au péril de sa vie. En effet, la pierre est censée préserver de la mort celui qui la porte :

Eracle a son seignor le livre,

Et li sergans quil tient por yvre

Vient se li dist: "Vous ne savés

De cest caitif que ci veés :

Por ceste piere c'ot veüe

Qu'eüst por sis deniers eüe,

Me fist livrer quarante mars !

Bien eust desservi qu'il fust ars !

Onques des bones cure n'ot,

Ne nule bargignier n'en volt ;

U nule bone n'en connut,

U le pior de gré eslut."

Li empereres s'en aïre,

Par maltalent li prist a dire :

“Por c'as tu la donné le mien,

Por la piere qui ne valt rien,

Et quant malvaise l'acatas

Quarante mars por l'en donnas?

Faire me valsis paringal

A ton signor le senescal

Qui por toi dona mil besans!

J'en sui certes grains et pesans!

- Ains devés estre liés, biaus sire,

Car bien l'os tesmoignier et dire

Que ceste piere valt tout l'or

Que vous avés mis en tresor,

Qu'eve ne fu n'arme ne crient

Ne ne puet cremoir qui le tient.

Se li caitis, li deceüs

N'eüst que sis deniers eüs

Se vertu perdist. - Puet cel estre?

-Por ce en donai tant au mestre.

Biaus sire, un seul petit m'oés :

Ardés m'en fu se vous poés,

Mais sor moi l'aie, el ne demant ;

U m'assaiiés d'arme trencant,

De toutes pars a moi lanciés

Et en m'aige me balociés :

S'a pieur vient, si soit sor moi.

- Eracle, voir, ensi l'otroi." 
Il ne se valt plus detrïer ;

Une grant muele fist loier

Entor Eracle le varlet ;

Se bone piere au col li met ;

Toute se gent i fait aler

Et si le fait adevaler

El Toivre qui est molt parfons

Et li muele le trait au fons ;

Une corde i ont atacie

A coi li muele ert sus sacie

Et li varlés u mors u vis.

Une grant liue, ce m'est vis

I a Eracles si geü

Qu'ils ne l'ont onques remeü,

Et je vous di que li pluisor

Deprient Diu le Creator

Par se merci qu'il le garisse

Que il en l'eve ne perisse.

"Folie, font il, vos travaille,

Qui cuide que prïere vaille!

Noiés est pieça et estains.

Grans biens seroit qu'il fust atains

Et c'on li fesist sepulture.

Mar fu si biele creature !

Il ne fu lere ne triciere

Ne baretere ne boisiere,

Mais por garir se mere, espoir

Dist qu'il estoit de tel savoir."

Molt en pleurent le gent menue ;

Cil ont le corde fort tenue

Si sont anuié del tenir

Et font il plus de gent venir

Por celui traire contremont,

Car li sire les en semont

Et reuve c'on le traie a fait

Por veoir con li cose vait

Et s'il est si con il a dit.

Trestout $\mathrm{i}$ vont sans contredit

Et traient fors a le polie ; [...]

A traire n'ont pas mis granment

Si le deslient erranment ;

Il saut en piés trestous delivres,

Or se tienent pluisor por yvres

De çou qu'il ont mesdit de lui,

Et pluisor voelent son anui

Et dient par lor legerie

Qu'il oeuvre tout par trecerie,

Par sorcerie et par enchant ; 
[...] Il est tous nus et tous descaus

Et moilliés, et li senescaus

Li giete au col un mantiel gris.

[...] Li empereres s'en sorrist,

Que il est molt de bon espoir

U cil l'a mis por dire voir ;

[...] Eracles l'a veü sourrire

Et dist : "Conment vous sanle, sire?

Ne sont mi dit bien veritable?

- Amis, s'il sont tuit si estable

Com a li premerains esté,

Rices sera ains cest esté.

- Sire, ostés vous de mescreance ;

Faites faire sans demorance

Un feu molt grant et molt plenier ;

Je ne lairai por nule denier

Que je n'i entre demanois..."

(Éracle, 886-1La séquence précédente s'achèvait sur un récit où un sergent racontait comment Éracle avait acheté une pierre d'apparence banale au prix d'une gemme précieuse. Il rapportait à l'empereur le prix exorbitant payé pour l'obtenir, information qu'Éracle avait négligée. Face à la colère de son maître, le jeune garçon s'engage à mettre la pierre à l'épreuve en se soumettant à une sorte d'ordalie de l'eau. La décision est prise au cours d'un dialogue. Pendant l'épreuve proprement dite, le héros - descendu dans le Tibre à la profondeur d'une lieue - n'est guère en position de parler; en revanche « la gent menue » se lamente sur sa disparition. Ce commentaire choral ne fait nullement avancer l'action. Qu'est-ce qui marque l'achèvement de ce processus ? On peut considérer tout d'abord la décision de l'empereur de faire ressortir Éracle de l'eau comme un véritable retournement de l'action. Cependant, il se situe dans la continuité de l'épreuve puisque celle-ci ne peut être considérée comme achevée avant que l'on ait pu vérifier que l'enfant vit encore.

Car li sire les en semont

Et reuve c'on le traie a fait

Por veoir con li cose vait

Et s'il est si con il a dit.

Cet ordre se contente donc de prolonger l'acte proprement dit. D'ailleurs son nivellement par le discours narrativisé est révélateur de son peu d'importance dramatique. De même les accusations désobligeantes des losengiers ne changent pas la situation. En revanche la discussion entre Éracle et l'empereur entérine la réussite du jeune devin. L'empereur la reconnaît officiellement par sa promesse d'une récompense, si les autres pouvoirs du garçon sont aussi visiblement prouvés. Ce dialogue sanctionne ${ }^{16}$ donc la fin de l'épreuve, qui n'est pas seulement la sortie de l'eau mais la reconnaissance de la vérité des propos d'Éracle. Seul un dialogue symétrique au premier, mettant en scène les mêmes agents, peut véritablement clore le processus. 
19 L'étude de cette scène permet donc de confirmer notre première conclusion : certains dialogues sont essentiels dans la construction des actions : celui où l'enfant s'engage dans l'épreuve, celui où l'épreuve est déclarée officiellement terminée. D'autres se limitent à la formulation illustrative des enjeux de la scène : les spectateurs commentent ce qui se passe, l'empereur prononce les ordres nécessaires au déroulement de l'épreuve.

\section{Les dialogues catalyseurs}

20 Les paroles des personnages permettent donc à l'action de passer d'un état d'éventualité à l'acte en engageant les personnages, et enfin d'achever cet acte en les désengageant ou en sanctionnant leur action. Le passage de l'éventualité à l'acte peut se faire selon deux modes : selon le mode de la motivation, comme dans les extraits que l'on vient de voir où les personnages décident de s'engager dans une action; ou selon le mode de la cause, où un autre engagement provoque une conséquence non désirée. Par exemple, dans la Fille du comte de Pontieu ${ }^{17}$, une série de mauvaises décisions entraîne le fatal isolement de Thibaut et de la dame dans une forêt mal famée : l'envoi par Thibaut de son chambellan en avant pour aller leur chercher une escorte et ensuite le choix de la " fause voie plus antee et plus large que la boine» que «les larons mibatoient pour faire les pelerins desvoier. » les laissent démunis et isolés dans un milieu hostile. Ces décisions sont prises au cours de petits dialogues qui marquent l'engagement tragique des personnages dans leur malheur. On est bien dans le cas de la cause, mais celle-ci reste du ressort de la responsabilité humaine. Et, en effet, il semble que dans ces récits médiévaux, les événements se rapprochent des actions et les causes des motifs. Dans cette même nouvelle, un événement comme la tempête qui emporte les personnages masculins en terre sarrasine est transformé en action par la prise de décision des personnages. Ils décident de gagner la première terre ferme qu'ils trouvent, quelle qu'elle soit, plutôt que de continuer à risquer leur vie en mer.

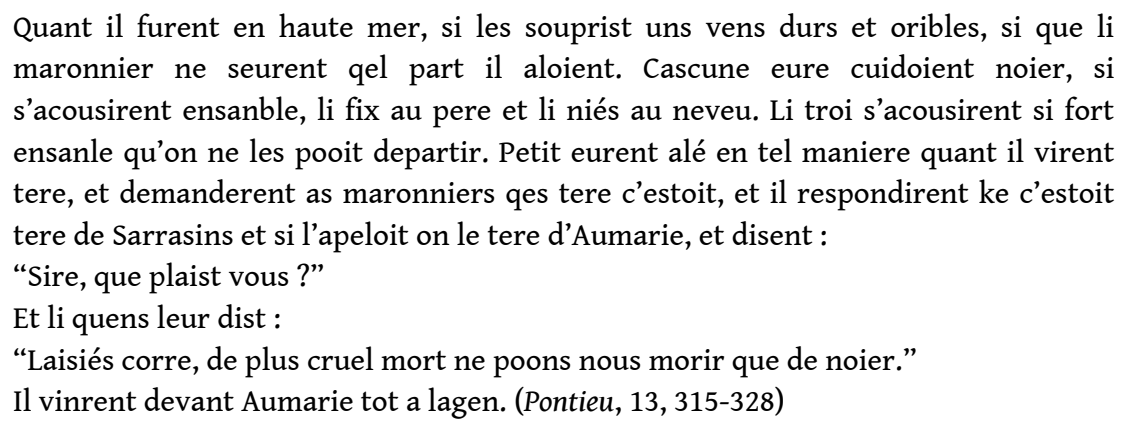

21 De patients subissant la tempête, ils deviennent agents, assumant la responsabilité de leurs choix. Le fait d'arriver en terre ennemie répond alors à une motivation (même faible: il s'agit avant tout d'éviter la noyade) et non plus seulement à une cause. Le dialogue permet donc d'engager l'action en y impliquant les personnages. Par leurs décisions, par leurs choix, ils deviennent partie prenante du sort que le narrateur 
leur réserve. La parole des personnages semble ainsi mettre en scène les réflexions médiévales sur le libre-arbitre. Le déterminisme du récit, l'omniscience narrative ne font pas disparaitre le « franc vouloir » que les personnages manifestent par leur parole. Quelle que soit la voie qu'ils choisissent, bonne ou mauvaise, c'est toujours eux qui finalement prennent les décisions qui influent sur leur avenir : "Lidée profondément ancrée dans les mentalités d'alors, de la puissance réelle de la parole engendre une vue morale de l'univers. Tout discours est action, physiquement et psychiquement effective. » remarque Paul Zumthor dans la Lettre et la Voix ${ }^{18}$. Mise en scène dans sa performativité, la parole engage moralement soi ou les autres.

Par quel biais se fait cet engagement dans l'action ? Il ne concerne pas seulement un individu volontaire et responsable de son propre sort, un personnage peut se trouver engagé malgré lui par un acte directif, dans une action dont il ne voulait pas, comme le malheureux Gahériet, obligé de se battre contre Tristan. Éracle s'engage par une décision qui prend aussi la forme d'un directif: "Ardés m'en fu se vous poés,/Mais sor moi l'aie, el ne demant; [...]/Et en m'aige me balociés ». Éracle étant un personnage socialement inférieur à son interlocuteur, il soumet sa décision à la permission de celui-ci : «Eracle, voir, ensi l'otroi. » lui répond l'empereur. Qu'il soit soumis à un ordre ou qu'il se décide volontairement, le personnage a besoin de la parole d'autrui pour accéder à l'action : ce qui provoque ce passage de l'éventualité à l'acte est un changement dans la modalité du /faire/. Le dialogue est le lieu où se réunissent deux modalités du /faire/, virtualisantes et actualisantes, et où elles débouchent sur une action, c'est-à-dire une modalité réalisante ${ }^{19}$. Éracle se limite à des modalités virtualisantes : un /vouloir faire/ et un / savoir faire/, que l'autorité de l'empereur, modalité actualisante de l'ordre du /pouvoir faire/ou du /devoir faire/, permet de transformer en modalités réalisantes, en /faire/ et en /être/. La jonction de ces éléments se fait non seulement dans le dialogue mais aussi par le dialogue dans lequel chaque personnage présente sa position. L'action de l'agent principal ne peut se déclencher que s'il réunit les modalités du /vouloir faire/, du /savoir faire/ et du /pouvoir faire/: «Labsence de l'une ou l'autre de ces modalités (auxquelles un /devoir faire/ peut être ajouté) débouche sur l'impuissance (non-pouvoir), l'apathie (non-vouloir), ou l'ignorance (non-savoir) ${ }^{20}$. » Le dialogue est le lieu où ces modalités sont «mises en commun », réunies pour permettre une "réaction ». Nous qualifierons de catalyseurs les dialogues qui, par la manière dont ils engagent les actants dans une action, permettent de faire basculer une action de l'éventualité à l'acte. Ce terme de catalyseur est inspiré de Roland Barthes ${ }^{21}$ qui distinguait deux fonctions dans un récit, les fonctions catalyses, qui « remplissent l'espace narratif » et les fonctions cardinales ou noyaux, qui constituent les véritables charnières du récit. Cependant, nous inversons ici le vocabulaire utilisé pour lui redonner ses connotations originelles. Là où Barthes parlait de catalyse "eu égard à leur nature complétive », nous choisissons de redonner à ce terme sa 
coloration métaphorique chimique : ce terme implique la modification d'un comportement sous l'effet d'un autre actant. Le dialogue catalyseur unit, rassemble des données qu'il fait réagir entre elles. Il produit un composé nouveau.

Ce phénomène d'engagement peut être rapproché du Contrat établi au début d'un récit entre le Destinateur-mandateur et le Sujet, selon la sémiotique narrative de Greimas. Le sujet a la capacité d'agir mais il est impuissant sans le Destinateur qui est celui qui «fait faire », "c'est-àdire qui exerce un /faire/ visant à provoquer le /faire/ du sujet ${ }^{22}$. » Toutefois, l'engagement que nous décrivons, s'il intègre ce contrat, ne s'y limite pas : alors que le Destinateur a un statut hiérarchique ${ }^{23}$ supérieur à celui du Sujet, les interlocuteurs qui s'engagent ou qui engagent autrui dans une action par un ordre (/devoir faire/) ou par un accord ou une permission (/pouvoir faire/), peuvent être des sujets situés au même niveau et qui risquent eux aussi leur être dans cette action. Ainsi le dialogue d'engagement peut être la Manipulation greimassienne, mais peut aussi être, plus simplement, une discussion qui aboutit à une décision mutuelle ou unilatérale. Par exemple, dans La Fille du comte de Pontieu $^{24}$, le débat entre les bandits qui discutent de ce qu'ils feront de la dame qu'ils ont capturée, assure le passage d'un /vouloir faire/ et d'un / pouvoir faire/ (quelque chose) à un /savoir (quoi) faire/, sans qu'aucun des bandits ne prenne une figure de Destinateur. Ils constituent tous ensemble un unique sujet de vouloir, doté de multiples voix, qui s'engage dans une décision.

L'analyse d'un extrait du Merlin ${ }^{25}$ nous permettra de décrire d'autres particularités de tels engagements :

Merlin demande à Uter, devenu roi après la bataille de Salisbury, ce qu'il compte faire pour honorer la mémoire de son frère qui y est mort.

Puis aprés une grant piece que Uitiers ot esté en son regne et tenu li en pais, si parla Merlins a lui et li dist :

"Coment? ne feras tu plus de Pandragon ton frere qui gist en Salebires?"

Et Uitierspandragons respont :

"Que veuls tu que je en face? Je en ferai quanque tu voudras et tu me loeras."

Et Merlins dit :

"Tu li juras et je li creantai que nos i feroions tel chose qui parroit tant com crestientez durroit. Aquite ton sairement, quar j'aquiterai bien ma parole."

Et Uitiers respont :

"Di moi que je puisse faire et et jel ferai molt volentiers"

Et il dist :

"Enprenons a faire tel chose qui i paire touz jorz ne jamés ne soit usee."

Et Uitiers dit :

"Jel ferai volontiers.

- Or envoiez donc por grosses pierres querre qui sont en Yrlande, si i envoierés vaisiaus, si les en fai venir, ne il nes savront ja si granz aporter que je nes dresce et je lor irai mostrer celes que je voil que il aportent."

Et Uitiers dit que il i envoiera molt volentiers; si i envoia genz et vaissias a molt grant planté. (Merlin 47, 24-42) vouloir faire/ et un /pouvoir faire/ sans objet. Il ignore sur quoi il peut les employer et les laisse dans l'oubli. Dans le dialogue, par les actes 
informatifs et directifs qu'il fait, Merlin lui rappelle son /devoir faire/ et lui apporte un /savoir faire/ qui oriente cette volonté pure, incapable de se mobiliser par elle-même. Merlin est ainsi celui qui oriente l'action par les informations qu'il fournit. Il lie la dimension pragmatique du /faire/ à sa dimension cognitive. La sémiotique appelle dimension pragmatique «l'univers de l'action proprement dite, mettant en scène des sujets humains avec des objets concrets (trésors cachés, territoires à conquérir, dangers à fuir, etc.) » et dimension cognitive celle qui porte sur des objets de savoir, le savoir étant considéré comme un objet de valeur dès qu'un acteur dispose d'une connaissance qu'un autre n'a pas :

Il suffit que deux acteurs dans un récit donné ne disposent pas d'un même savoir sur les objets pour que ce savoir devienne un objet de valeur (secret, illusoire, mensonger, vrai), et donc un enjeu narratif. Centrée sur l'excroissance de ce paramètre modal, la dimension cognitive s'est déployée dans les récits littéraires ${ }^{26}$.

La circulation de cette connaissance fonctionne comme un enjeu narratif qui fait progresser l'action. Le /faire/ cognitif se partage en deux pôles : le /faire/ persuasif et le /faire/ interprétatif ${ }^{27}$. Dans cet extrait de Merlin, comme dans celui d'Éracle, l'un des deux personnages se caractérise par un faire persuasif fort et domine l'autre. Cependant, une action peut s'engager, comme on le verra plus bas, simplement sur le /faire/ interprétatif de l'un des agents analysant une situation.

Les dialogues qui engagent l'action peuvent porter uniquement sur la dimension pragmatique, par exemple l'ordre de se battre, ou plus fréquemment comporter aussi une dimension cognitive, par exemple l'information sur les dons de la pierre qu'Éracle révèle à l'empereur. Dans ce dernier cas, le savoir précède et détermine le vouloir. Les dialogues catalyseurs vont donc rassembler actes informatifs (le /savoir ou pouvoir faire/), actes directifs ou promissifs (/devoir ou vouloir faire/) :

Après avoir vu les noms de leurs camarades sur le tombes du cimetière de la Douloureuse Garde, Gauvain et ses compagnons quittent la citadelle. Mais le vavasseur hospitalier qui se propose pour les héberger est un traitre qui va les emprisonner.

Et lors avalent jus do chatel por aler herbergier, si encontrerent un vavassor meslé de chienes qui mout sanbloit estre preudome. Cil demanda a monseignor Gauvain qui il estoit.

"Por qoi lo demandez vos? fait messires Gauvains.

- Sire, fait il, ge nel demande se por vostre preu non, ce sachiez.

- Et gel vos dirai, fait il, car bien sanblez preudome. Ge sui Gauvains."

Et qant li vavassors voit les lermes qui ancor li chaoient des iauz, si li demende por qoi il plore. Et il dit qu'il plore por la mort des compaignons lo roi qu'il a veüz laïssus en cel chastel.

"Sire, fait il, or ne vos dolosez mie tant, tant que vos sachiez por quoi. [...] Por ce lo vos lo que vos en veigniez o moi herbergier anuit mais et tant com vos demorroiz en cest païs. [...] Et sachiez que li plus de ce que vos avez veü laïssus n'est se mençonge non et anchantemenz. Mais ge vos mosterrai verité, car ge vos ferai veoir des compaignons lou roi une partie toz sains et toz vis de cex que les letres laïssus tesmoignent a mort."

Quant messires Gauvains l'ot, si en est trop liez. Et dit que dons ira il, car il n'est nule terre o il n'alast por tant de prodomes veoir. Li vavassors s'an ala avant et li dis compaignon aprés. (Lancelot ${ }^{28}$, p. 548-550) 
Ce dialogue qui engage l'action «capture de Gauvain et de ses compagnons » n'a rien d'original : il est construit sur le passage de l'information à l'action. Il commence par deux séries de questions du vavasseur, d'abord sur l'identité puis sur le comportement de ses interlocuteurs. Les informations reçues vont lui permettre de nouer le piège qu'il prépare: sa vengeance contre le roi Arthur ne peut mieux s'exercer que contre son propre neveu et les révélations sur les tombes maquillées du cimetière de la Douloureuse Garde fournissent un prétexte idéal pour l'attirer chez lui. L'échange didactique «Por qoi lo demandez vos ? fait messires Gauvains.- Sire, fait il, ge nel demande se por vostre preu non, ce sachiez », enchâssé dans ces informations, apporte d'abord un effet de réel mimétique en cassant le rythme linéaire questions/ réponses, donne ensuite une profondeur psychologique à Gauvain qui ne livre pas d'emblée son identité et, surtout, permet enfin d'insister sur le caractère mensonger des propos du vavasseur. Par deux fois, il aura été fait mention de sa «semblance » de prud'homme, mention qui ne peut manquer d'alerter un lecteur averti. Le dialogue se poursuit par un échange promissif: [offre/accord], par lequel chacun des locuteurs s'engage. Le passage d'une partie didactique à une partie promissive permet donc le passage d'un /savoir/ à un /vouloir faire./ Ce dialogue un peu développé présente la forme la plus classique de construction d'un dialogue catalyseur, il n'intègre guère de surprises ni de péripéties.

De petits dialogues peuvent être groupés pour constituer le même effet catalyseur. La partie informative et la partie promissive se trouvent alors séparées dans deux échanges différents :

Tristan prend la décision de combattre avec Dynadan contre trente chevaliers qui ont préparé un guet-apens contre Lancelot. C'est une demoiselle qui leur révèle ce piège.

Quant mesire Tristans vit la damoisele plourer si tenrement, il s'arestut tout maintenant en mi le cemin et le mist en teus paroles com avoient fait li autre compaingnon. Et tant fist a la damoisele, et par proiieres et par manaches, qu'ele li dist l'ocoison de son doel tout autresi conme ele avoit fait as .IIII. compaingnons. Quant ele a son conte finé, et ele li a devisees les paroles que je vous ai cha ariere conté autre fois, mesire Tristans li dist adonc:

"Madamoisele, or vous em poés vous hui mais aler quel part que vous vaurés. Je vous conmant a Nostre Signeur. Or sachiés que je me tieng a bien paiié de ces nouveles. Beneois soit Diex qui soufri que je vous trouvaisse a cestui point pour oïr la verité de ceste cose !"

Lors s'en vait mesire Tristans un poi avant et dist a Dynadant :

"Dyanadant, que ferom nous? Il n'a mais fors de l'aler. Se Diex nous veut aidier, hui en cest jour nous avenra si grans hounours que onques a nul jour graindre ne nous avint. Car chertes il est mestier que nous metom a mort $u$ a destrusion tous ces .XXX. cevaliers ki Lanselot du Lac voelent metre a mort. Je ferai d'aus, se Diex le me veut consentir, ce k'il voelent entr'aus faire de chel preudoume."

Atant se metent a la voie sans faire autre delaiement. (Tristan 2, 26, 26-38; 27, 1-10)

Deux petits dialogues se suivent, le premier, mis très en retrait, constitue la partie informative, le second la partie promissive. Les actes de langage informatifs ne peuvent fonctionner seuls, ils doivent être accompagnés d'un acte de langage directif ou promissif pour prendre une valeur « engageante ». Leur présence permet cependant de relier les 
événements précédents avec ceux qui vont suivre. En ce sens, ils ont véritablement pour fonction de nouer différentes lignes narratives et d'en faire naître une nouvelle. Ainsi, ils ont souvent une fonction rétrospective, analeptique, ou bien ils ouvrent sur des événements "simultanés », non encore connus de l'un des personnages ou du lecteur. Ils permettent d'enchaîner les actions des uns et des autres et de donner à cet enchaînement un tour logique. Ils créent ainsi des liens avec des événements qui se situent dans un autre espace-temps diégétique. Dans les romans en prose cycliques, qui se caractérisent par leur volonté totalisatrice de saturer l'espace romanesque, ils peuvent, de ce fait, contribuer à faire naître un univers complexe et complet. Danielle Coltier voyait là une fonction distincte de cohésion, il s'agit essentiellement, pour nous, du corollaire de leur fonction dramatique. Pour assurer leur fonction d'engagement, les dialogues catalyseurs ont besoin d'intégrer une partie informative qui contribue à créer un réseau narratif avec les autres actions du récit.

\section{Les dialogues analyseurs}

31 Inversement, nous qualifierons d'analyseurs les dialogues qui ne font pas basculer l'action dans l'une ou l'autre de ses étapes. Ces dialogues peuvent être supprimés de l'enchaînement des actions car ils n'apportent rien à la construction de l'intrigue, mais ce sera au prix d'une déperdition-plus ou moins importante-du sens de celle-ci. Leur fonction n'est pas dramatique mais essentiellement sémantique : elle construit du sens. Le dialogue analyseur décompose, sépare les divers constituants d'une action en explicitant ouvertement les enjeux qui la sous-tendent, et ce faisant, il l'éclaire, l'explique. Les commentaires de la « gent menue » sur le triste sort que subit le jeune Éracle disent l'aspect pathétique de la situation ; les sarcasmes que se jettent à la figure Tristan et Palamède remplacent et symbolisent pour l'auditeur-lecteur les coups qu'ils se donnent. La violence du combat en est augmentée cependant que l'intensité de leur haine est mise en valeur. Ces dialogues permettent au narrateur de montrer une situation plutôt que de la dire, comme si «l'histoire se racontait elle-même ${ }^{29}$ » selon l'injonction bien connue des romanciers anglo-saxons habitués à opposer le showing au telling. Ils contribuent donc au sens du récit, moins par l'apport d'explications que par la redondance qu'ils constituent par rapport au discours. Un certain nombre de dialogues conclusifs limités à l'évaluation de l'action achevée sont, en ce sens, des analyseurs. Ils rendent explicite le sens que le lecteur attentif avait déjà dégagé. Comme le dit $R$. Barthes, qui, rappelons-le, les désigne sous le nom de catalyse, cette fonction constitue les expansions des noyaux qui engendrent l'histoire :

[Elle] réveille sans cesse la tension sémantique du discours, dit sans cesse : il y a eu, il va y avoir du sens; [sa] fonction constante est donc, en tout état de cause, une fonction phatique (pour reprendre le mot de Jakobson) : elle maintient le contact entre le narrateur et le narrataire. Disons qu'on ne peut supprimer un noyau sans 
altérer l'histoire, mais qu'on ne peut non plus supprimer une catalyse sans altérer le discours ${ }^{30}$.

Les dialogues analyseurs viennent illustrer l'action en cours, ils l'accompagnent sans la faire : ils se contentent de la répéter sous une autre forme. Cette illustration comporte une dimension iconique, symbolisante, qui met en valeur un détail pour renvoyer à un ensemble plus vaste de significations. Ils ont un rapport métonymique avec l'action et fixent ce que le récit annonce.

Enfin ces dialogues contribuent à la mise en place du système de valeurs $\mathrm{du}$ récit. Consistant souvent en des commentaires sur l'action, ils construisent un jugement sur ce que vivent les personnages. Ils permettent ainsi de hiérarchiser les actions et par conséquent, de classer les personnages en «bons» ou «mauvais», «fortunés» ou « infortunés ». Petit à petit s'édifie ainsi concrètement la réputation, le pris et le los, des chevaliers arthuriens. La rumeur qui se répète et grossit autour d'eux symbolise et figure à la fois leur renommée grandissante.

Les dialogues analyseurs ne sont pas variés, ils consistent souvent en commentaires des protagonistes ou des spectateurs de l'action. Mais l'illustration peut aussi venir d'actes de langage plus contraignants auxquels, pour une raison ou pour une autre, la valeur dramatique est retirée.

Avec l'arrogance de la jeunesse, Lancelot, qui voit que son propre adversaire est sur le point de céder, propose à Keu d'échanger leurs combats.

Si dure mout la bataille d'aus deus, tant que li chevaliers nel pot soffrir, si guerpist place plus et plus et cil prant terre sor lui. [...] Et bien voient qu'il en a mout lo poior et que trop est au desouz. Et entre Keu et lo suen chevalier orent lor chevaus ocis et furent a pié. Et li noviaus chevaliers redit :

"Venez ça, sire Kex, car vos veez bien comment il est ; et vos me laissiez celui, car j'ai autre chose affaire que ci demorer tote jor."

Et Kex en a mout grant honte, si li dit par corroz :

"Biax sire, bien vos coviegne del vostre, et lo mien me laissiez."

Et lor recort li noviax chevaliers sus au suen chevalier... (Lancelot, p. 488)

Le refus du sénéchal oblige Lancelot à continuer et à faire durer son duel (il se met à ménager son adversaire pour ne pas gagner trop vite !) Cet acte directif aurait engagé les personnages dans une nouvelle action (combat contre un autre adversaire). Refusé, il se contente d'illustrer la force hors du commun du jeune chevalier, meilleur jouteur que le sénéchal du royaume.

Dans l'Escoufle ${ }^{31}$, la recherche des deux jeunes héros poursuivis par les messagers de l'empereur est symbolisée par les interrogatoires auxquels sont soumis les gens que ces messagers rencontrent, mais les réponses négatives qu'ils reçoivent montrent que nul ne sait où sont passés les enfants. Une réponse informative aurait orienté tout de suite l'action vers une piste ; l'absence de toute donnée illustre la réussite de leur fuite et leur habileté :

Li un s'en sont alé vers Genvres

Ou Guilliaumes fu norris jenvres,

Li autre en Sezile et en Pulle,

Ml't emportent avoir a pulle ; 


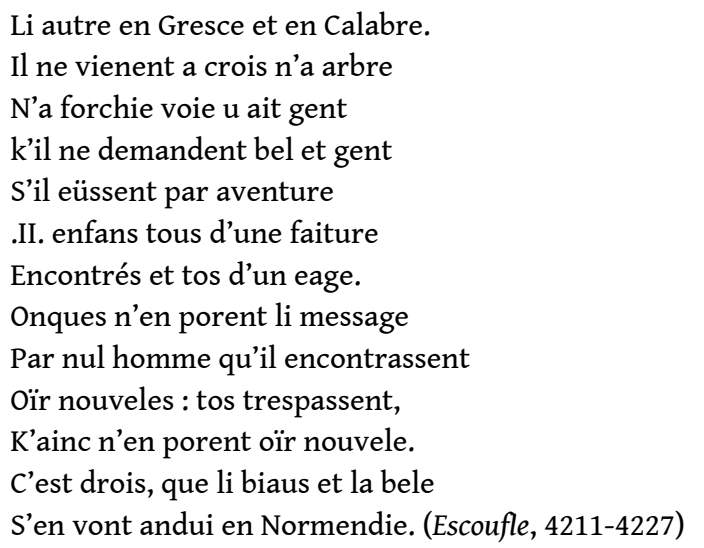

Les échanges didactiques vains auxquels sont condamnés les messagers empêchent l'action de basculer et signalent l'absence de tout indice. Ils viennent appuyer le discours du narrateur sur la discrétion de Guillaume et d'Aélis. Les dialogues illustratifs sont le plus souvent schématiques, ils dépassent rarement trois répliques et sont le lieu privilégié des voix chorales qui commentent l'action dans des répliques isolées. Si les échanges sont complets, il s'agira le plus souvent d'échanges de type [commentaire/commentaire]. On peut cependant y trouver, comme nous venons de le montrer, d'autres types d'échanges qui ont perdu leur fonction d'engagement.

\section{Les dialogues d'achèvement}

Les dialogues qui achèvent une action sont plus difficiles à analyser ; ils mêlent en effet divers types de comportements et forment une catégorie mixte : plutôt catalyseurs s'ils consistent essentiellement en un désengagement d'un locuteur de l'action, ou plutôt analyseurs s'ils consistent en une évaluation de cette action. Par exemple, lors d'un combat du tout jeune Lancelot contre des chevaliers, la volonté de l'un des protagonistes de se retirer suffit à mettre fin à cette action :

Qant li chevaliers qui l'avoit navré oï qu'il disoit que l'an les li laissast andeus, si commence a rire. Lors vient a lui, et si li dit :

"Biax sire, certes ancor vos baillerai ge la moie espee se vos volez, ne a vos ne me combatrai ge mais hui.

- Ne ge, voir, fait li autres chevaliers.

- Par Sainte Croiz, fait li vallez, dont quiteroiz vos la pucele.

- Nos la vos quitons, font il endui, et savez vos por quoi? Nos veons bien que vos iestes de trop haut cuer, si poez encores venir a mout grant chose; et vos iestes si navrez que bien en porriez morir, se encores estiez un po grevez. Por ce si vous avons faite ceste bonté.

- Moi ne chaut, fait li vallez, por quoi vos l'aiez fait, mais que la pucelle soit quitee.

Or la me bailliez, car ge la voil.

- Volentiers", font li chevalier.

Li uns trait une clef, si la giete el prael et li dit :

"Damoisele, desfermez cele nef et venez hors, car cist chevaliers vos a conquise."

Cele defferme une nef qui el prael estoit atachiee a une chaine, puis est hors venue. Et li dui chevalier qui la gardoient s'an partent et s'an vont a lor affaire. (Lancelot, pp. 474-476)

La fin de l'action « combat » se manifeste par la décision des deux adversaires de Lancelot de cesser de se battre: «ne a vos ne me combatrai ge mais hui. - Ne ge, voir, fait li autres 
chevaliers. » Elle est issue du /faire/ interprétatif des deux personnages, qui ont réfléchi sur le comportement de Lancelot, et débouche sur une modification de leur /faire/ pragmatique: "Nos veons bien que vos iestes de trop haut cuer, si poez encores venir a mout grant chose; et vos iestes si navrez que bien en porriez morir, se encores estiez un po grevez ». Un refus de se désengager de l'un des trois combattants aurait prolongé l'action.

Quelle est la relation entre cette action et la suivante? La décision des deux chevaliers entraine l'action suivante : celle de libérer la jeune fille, action subordonnée et beaucoup moins importante, qui ne comprend aucun dialogue en dehors de l'ordre donné à la jeune fille de détacher sa barque. Le catalyseur qui actualise cette libération est l'ordre donné par Lancelot qu'on lui confie la jeune fille: « mais que la pucelle soit quitee. Or la me bailliez, car ge la voil. » En revanche, l'ordre donné à la jeune fille par l'un des adversaires du héros n'est qu'une illustration de cette action : «Damoisele, desfermez cele nef et venez hors, car cist chevaliers vos a conquise. » Ce deuxième ordre répète la décision initiale de Lancelot, qui, seule, a mis en route l'action de libération. Il est plus symbolique : il dit que la libération est en train de s'effectuer. Nous pouvons en conclure que le dialogue qui marque l'achèvement du combat par le désengagement des combattants est aussi celui qui marque l'engagement dans une nouvelle action, la libération. Le désengagement consiste en un abandon du /vouloir faire/, qui se trouve par conséquent déplacé sur une autre action.

41 Cet abandon du /vouloir faire/ peut se manifester non seulement dans le dialogue quand un personnage annonce la décision qu'il vient de prendre - il pourrait en effet se manifester autrement, par une fuite par exemple - mais aussi par le dialogue, quand, dans le processus de la discussion, les personnages sont amenés à abandonner leur / vouloir faire/ sous l'effet d'une nouvelle information: ainsi les combats qui opposent Kahédin et son père, ou encore Lancelot et Néronneus dans le Tristan s'achèvent-ils de la même façon sur des dialogues qui commencent de manière informative - l'un des deux protagonistes veut en savoir plus sur son adversaire - et se terminent sur un acte de langage promissif : la volonté de ne plus se battre. Le cheminement de l'information, du cognitif, à la décision, au pragmatique, entraîne le passage d'un /savoir (être)/ à un / vouloir ne plus faire/.

Dans l'extrait d'Éracle, précédemment analysé, les dernières répliques renvoient au début $\mathrm{du}$ processus, à l'affirmation que la pierre était magique : « Conment vous sanle, sire ?/ Ne sont mi dit bien veritable? » L'empereur est obligé d'acquiescer. Le dialogue d'achèvement vient donc apporter une réponse à la question posée dans l'engagement. Éracle propose ensuite de tester le pouvoir de la pierre en se faisant mettre au bûcher, ce qui l'engage dans une nouvelle action.

43 On peut, à partir de cette première conclusion, avancer dans notre analyse des dialogues d'achèvement en les comparant avec la Sanction et la Rétribution qui closent le récit (populaire) selon Greimas, comme nous l'avions fait par la comparaison entre la Manipulation et le dialogue catalyseur. Selon Greimas, la Sanction consiste en la reconnaissance d'un acte accompli :

Celle-ci peut être positive (gratification) ou négative (réprobation), pragmatique (récompense ou punition) ou cognitive (éloge ou blâme). [...] Le Destinateur[judicateur], en fin de parcours, vérifie la conformité de l'action accomplie au regard des termes de l'engagement, rétribue ou punit apportant ainsi lui-même sa contribution au contrat initialement conclu ${ }^{32}$. 
44 conforme au mobile qui l'avait initiée. Il porte un jugement de valeur à son sujet : ainsi Dynadan reconnaissant la capacité de Tristan à se battre encore : "Et voirs est, je le connois bien. Encore n'estes vous pas a che menés que je quidoie. »; ainsi Kahédin et Néronneus demandant pardon à leur adversaire pour avoir osé combattre contre eux, tandis que leur valeur guerrière est reconnue par ces mêmes adversaires. Mais, de même que le dialogue d'engagement ne se superpose pas directement à la Manipulation, de même le dialogue d'achèvement ne peut être purement et simplement assimilé à la Sanction parce que l'évaluation portée sur l'action n'est pas forcément issue d'un Destinateur, "souverain à la manière de Mithra, gardien des contrats, de l'équité des rapports

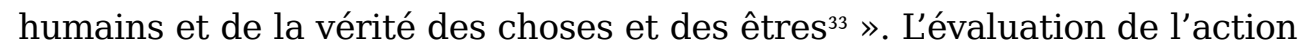
accomplie se fait au sein du dialogue, par les actants qui y ont participé, sans qu'aucune contrainte hiérarchique ne s'y ajoute :

Après lui avoir expliqué les raisons prophétiques qui empêchaient sa tour de tenir, Merlin quitte Vertigier, satisfait du travail accompli.

Ne Merlins ne fu mie iluec [a Wincestre], car si tost com il ot dit au roi por quoi sa tor chaoit et la senefiance des .II. dragons, si prist congié, si s'en ala et dist que il avoit bien fait bien ce por quoi il estoit venuz. Et einsis s'en ala en Norhumbellande a Blaises et li conta les choses, et Blaises le mist en son livre et par son livre le resavons nos encor. (Merlin, 31, 7-13)

Dans cet extrait, la première parole de personnage est un commentaire non adressé. Son isolement renforce son aspect conclusif. Un jugement axiologique est porté sur l'action terminée, le désengagement ayant été marqué par la prise de congé. Ce « mot de la fin » n'a d'autre fonction que de marquer l'achèvement de «la séquence Vertigier», il permet d'organiser le récit, d'en marquer les grandes unités. Mais nous ne croyons pas, comme Danielle Coltier, que ce rôle de transition soit une sorte de justification a posteriori de la structure du roman. Il n'est possible que parce que cette parole s'inscrit dans le processus d'achèvement d'une action. C'est parce que certains dialogues ont d'abord une fonction d'achèvement ou d'ouverture qu'ils peuvent ensuite gérer l'organisation logique des scènes au sein du récit. La fonction de régie du dialogue découle de son inscription dans l'action.

Ici aucun Destinateur ne vient évaluer l'action accomplie, parce que Merlin, rejeton du diable racheté par Dieu, n'en a pas besoin et qu'il est à la fois sujet et destinateur de son action. Nous conclurons de manière plus simple que l'achèvement dialogué d'une action peut comporter une évaluation de cette action, mais n'émane pas forcément d'un personnage en position de supériorité hiérarchique.

Dans un dialogue d'achèvement, les actes de langage et les enchaînements qu'ils constituent vont varier selon qu'il est plutôt catalyseur ou plutôt analyseur. Lévaluation se manifestera par des jugements qui donneront du sens à l'action menée ou par des actes de langage expressifs qui complimentent, blâment ou remercient. Le désengagement et/ou l'engagement dans une nouvelle action sera le fait 
d'actes de langage promissifs ou directifs. La part des uns et des autres variant selon la fonction dominante du dialogue. Le verdict du juge dans Merlin, à la fin du procès de la mère donne une place importante, quoique subordonnée, à l'évaluation :

Einsis se départent de ce consoil et vinrent devant le pueple, et li juges dist :

"Cist a bien sa mere rescousse, par raison, d'ardoir et saichent tuit cil qui le voient par le mien esciant qu'il ne verront jamés plus saige home."

Et cil responnent :

"Diex en soit loez, s'ele est respitee de mort." (Merlin, 15, 39-44)

Lénoncé du juge est déclaratif, il formule publiquement le verdict de ce procès hors du commun. En même temps, il l'accompagne d'un jugement valorisant à l'égard de Merlin. La foule répond par une formule d'action de grâce expressive. Cette fois, l'évaluation est nette dans des actes de langage orientés vers le commentaire.

Nous dirons donc que les dialogues d'achèvement, plus rares que ceux d'actualisation d'une action, consistent en un désengagement par l'abandon du /vouloir faire/, à la suite ou non d'une information. Ils comportent en outre une évaluation de l'action accomplie qui peut être le fait des protagonistes eux-mêmes. Ces dialogues sont des catalyseurs en ce qu'ils modifient une situation par l'action de l'un des agents sur l'autre. Ils produisent une situation nouvelle, issue de la réaction de l'agent concerné. Inversement, quand ils se limitent à son évaluation, ils ne sont plus indispensables à l'achèvement du processus, ils se contentent de le souligner, mais contribuent à lui donner un sens. Ils seront alors plutôt analyseurs. Ces deux catégories renvoient, d'une manière différente mais convergente, à la fonction que Danielle Coltier qualifiait de cohésion. Ces dialogues sont rarement autonomes et le plus souvent, une partie du dialogue sert de catalyseur à l'action suivante.

\section{Le dialogue considéré lui-même comme action}

À l'intérieur de ces catégories, quelques dialogues, particulièrement développés par leur longueur, particulièrement complexes dans leur structure, dans les enchainements d'actes de langage sur lesquels ils reposent, méritent une attention plus précise. S'ils peuvent être qualifiés de catalyseurs ou d'analyseurs à l'échelle du roman, ils adoptent à leur propre échelle la forme d'une action à part entière dont ils possèdent toutes les dimensions. Nous les qualifierons de dialogues-scènes.

Jusqu'ici nous avons considéré l'action sous son aspect pragmatique, matériel, voire physique. Mais un certain nombre d'actions n'ont qu'un aspect langagier et ne dépendent que de la dimension cognitive du récit: rien ne se passe en dehors de l'échange des propos. L'action, entièrement concentrée dans l'interaction verbale entre les personnages, se limite à un faire cognitif d'une importance considérable. Le dialogue théâtralise l'action qui prend une valeur spectaculaire, ou exalte le pur jeu social du parler. Si une conversation peut être considérée en elle-même comme une action, peuton faire coïncider les différentes étapes du dialogue avec celles d'un processus?

Pour que le dialogue-scène commence, il faut, comme dans toute action, que les personnages s'y engagent et opèrent une combinaison des modalités virtualisantes du / faire/ avec les modalités actualisantes : cela passe essentiellement par la valorisation de l'objet de la conversation qui y est érigé en bien à obtenir. L'engagement des personnages 
dans la parole est provoqué par la transformation de la conversation en objet de valeur, suffisamment motivant pour constituer un /vouloir faire/.

Dans l'Escoufle ${ }^{34}$, le récit des aventures de Guillaume constitue un noyau de l'intrigue, qu'il fait basculer dans sa résolution, en ce sens le dialogue pris dans son ensemble peut être vu comme catalyseur, mais, considéré en lui-même comme une action, il peut être décrit selon les diverses étapes que l'on a repérées. L'action peut être résumée ainsi : Guillaume raconte ce qui lui est arrivé. Elle a des agents: le narrateur Guillaume et ses auditeurs, parmi lesquels, incognito, Aélis, sa fiancée, dont il a été malencontreusement séparé et qu'il cherche depuis plusieurs années; elle se poursuit dans un cadre, une veillée seigneuriale ; elle a des motifs : les auditeurs veulent se divertir d'une histoire étonnante, Guillaume veut gagner de l'argent pour pouvoir continuer à chercher Aélis; elle a enfin une issue: les retrouvailles des amoureux, issue qui consiste en un renversement de situation, puisque ceux qui étaient séparés sont réunis, ceux qui étaient déshérités retrouvent une situation sociale. Le récit de Guillaume est d'abord éventuel tant qu'il n'est pas en train de le raconter. D'ailleurs, il risque à deux reprises de ne jamais commencer, car le héros se fâche et cherche à quitter la pièce. La première partie de cette scène a donc une fonction de catalyseur, puisque les autres personnages veulent engager le jeune homme dans l'action. En effet, ils l'ont vu attraper et dévorer un milan de façon particulièrement sanguinaire et aimeraient comprendre le sens d'un tel geste. L'information y est érigée en objet de valeur pour ses auditeurs par la curiosité qu'a suscitée son comportement et est d'ailleurs directement monnayable : le comte propose de le payer trente marcs pour son récit. Pour le héros, la possession de ce secret, symbolisée par la conservation, malgré les vicissitudes, de l'aumônière et de l'anneau qu'Aélis lui avait confiés, représente sa dernière possession, la dernière trace de son identité. Il lui est donc difficile de la donner en pâture à un public.

Dans le Tristan en prose, la rencontre de Lancelot, Palamède et Kahédin, dans l'obscurité de la nuit, autour d'une source, est entièrement une scène. Ce dialogue ne catalyse aucune autre action, puisqu'au petit matin, les chevaliers se préparent à se séparer, il est plutôt analyseur dans la mesure où il commente les amours malheureuses des chevaliers. Il suit un monologue pathétique de Kahédin, écouté silencieusement par les deux autres chevaliers restés dans l'ombre, dans lequel celui-ci déplore son amour sans espoir pour la reine Yseut. L'ensemble constitue une pause dans la suite de joutes et de rencontres qui jalonnent la vie des chevaliers errants. La discussion commence par ce moment de bascule où les auditeurs indiscrets s'engagent à parler :

Palamidés, ki plus estoit un petit hastis de parler que n'estoit Lanselos, quant il entent que Kahedins aloit ainsi parlant, il se tourne tout maintenant sour son escu et s'asiet en son seant. Quant Kahedins l'ot remuer, il laisse esranment sa complainte et le conmence a regarder, et tant le regarde ententivement par mi l'oscurté de la nuit k'il connoist tout certainnement que ce est chevaliers armés. Lors parole esranment et dist :

"Ki estes vous la?"

Palamidés respont atant et dist :

"Vassal, je sui uns cevaliers, ki ai vos paroles escoutees que je tieng a mout grant merveille. Mout vous ai oï blasmer Amours et dire paroles encontre li. [...]."

Quant Palamidés a parlé, Kahedins li respont atant et dist :

“Dans cevaliers, se Diex m'aït, se je quidaisse que vous fuissiés si pres de moi conme vous estes, je n'eüsse pas dit de teles paroles ai je dites. Nonpourquant, puis que je l'ai dit, je le conferme et di bien tout apertement..." (Tristan 1, 101, 10-30) 
55

début de dialogue est bien catalyseur. Par la question qu'il pose Kahédin engage son interlocuteur. La nature directive de la question oblige l'autre à répondre. Palamède est contraint à un /devoir parler/ qu'il prolonge en /vouloir parler/. En effet, il pourrait se contenter de signaler sa présence et de s'excuser d'avoir écouté des propos qui ne lui étaient pas destinés. La conversation ne passerait alors pas à l'acte. Au contraire, il porte un jugement sur ce qu'il a entendu, obligeant ainsi son adversaire à réagir. Kahédin a, lui aussi, le pouvoir de ne pas poursuivre le débat qui s'engage. Malgré le désagrément provoqué par le fait de se sentir observé, il confirme ses propos et lance véritablement le dialogue. Chacun des locuteurs s'est engagé, est passé d'un /pouvoir parler/ à un / vouloir parler/.

L'entrée en jeu de Lancelot se fait de la même manière :

Aprés ceste response ne se puet plus tenir Lanselos de parler : tant a oï et entendu k'il vaura parler des ore mais. Il s'estout tout premierement, por ce k'il le sacent illuec. Palamidés, ki encore ne s'estoit pris garde de lui, quant il l'avise et il voit k'il est si pres de lui, il li dist adont:

"Vassal, ki estes vous, ki avoec nous estes ne nous dites nule parole ? Longement vous estes ore celés! Le faites vous pour paour de nous u pour mal que vous nous voelliés, ki ne nous avés encore riens dit ?" (id., 107, 1-9)

Lancelot a un /vouloir parler/ mais il lui est encore délicat d'intervenir dans la conversation qu'il écoute en silence. Un signe physique est nécessaire. Son petit toussotement, qui signale sa présence, donne l'occasion à Palamède de l'interpeller et lui permet de transformer son / vouloir parler/ en /pouvoir (devoir) parler/.

Dans le Merlin, le devin, qui s'apprête à révéler à Uter les secrets des Tables de la Cène et du Graal, n'engage pas la conversation avant d'avoir posé un certain nombre de conditions d'écoute au roi :

Si le traist un jor a consoil, si li dist :

"Sire, il me convendroit que je me descouvrisse a vos dou plus haut consoil que je sache, quar je voi que vostre terre est si en pais et que vos estes si bien sires de vostre regne que nus hom ne porroit mielz estre sires dou suen. Et por ce que je vos aim et por ceste chose que je vos voil dire vos sauvai je, quant Engis vos dut ocirre, quar il m'estoit bien avis que vos me devoiez molt croire et amer."

Et li rois respont :

"Il n'est nule riens que tu me voilles dire dont je ne te croie et que je nou face a mon povoir."

Et il respont :

"Sire, se vos le faites, le preu en sera vostre, quar je vos enseingnerai a faire tel chose qui gaires ne vos grevera ne vos ne savroiez ne porroiez faire nule chose par quoi vos eussiez si legierement l'amor de Dieu."

Et li rois respont :

"Di seurement, quar tu ne diras ja chose, s'ele puet estre faite, que je ne la face."

Lors dist Merlins :

"Ce vos sera molt estrange chose que je vos dirai et je vos pri que vos le celez et que vous nou dites mie au pueple ne a vos chevaliers; quar je voil que le preu et l'onor et le gré Nostre Seingnor en soit vostre."

Et li rois li creante que il n'en parlera se par lui non. (Merlin, 48, 4-26)

Merlin commence habilement, par des annonces valorisantes «dou plus haut consoil que je sache» ou "nule chose par quoi vos eussiez si 
legierement l'amor de Dieu » destinées à susciter chez son interlocuteur un /vouloir parler/ ou plus précisément, vue la relation inégalitaire qui caractérise ces deux personnages, un /vouloir écouter/. Comme pour une requête de don en blanc, il obtient un engagement d'écoute et d'obéissance absolue, répété à plusieurs reprises : "Il n'est nule riens que tu me voilles dire dont je ne te croie et que je nou face a mon povoir. » ou «tu ne diras ja chose, s'ele puet estre faite, que je ne la face. » Une dernière condition, la promesse du secret, constitue le dernier acte promissif permettant à la conversation de s'engager pour de vrai. Il lui révèle alors toute l'histoire des tables sacrées, dont la Table Ronde sera l'aboutissement.

Le corps du dialogue est l'action elle-même, sans qu'il y ait de relation d'illustration ou de redondance entre ce qui est dit par les personnages et par le narrateur. Celui-ci s'efface pour leur laisser les mains libres. L'enjeu de cette action sera un faire cognitif, didactique dans le cas de Merlin et d'Uter ou de Guillaume et du comte de Saint-Gilles : il y a un simple partage de connaissances.

61 Cependant un dialogue polémique peut aussi relever de cette dimension cognitive : dans l'exemple du Tristan en prose, la discussion qui oppose Kahédin à Palamède, que l'on pourrait rapprocher des batailles de clercs, oppose deux faire interprétatifs. Les chevaliers disputent sur l'identité de la femme décrite par Kahédin : "Et coment savés vous, fait Kahedins, que je aim cele que vous amés ? Ja ne vous ai je mie dit encore son non. » (102, 6-8), et sur la qualité de l'amour qui lui est porté... La qualification de l'objet dont Kahédin parle constitue l'enjeu de la discussion. L'entrée en jeu de Lancelot oriente la discussion vers une valeur métacommunicative : est-il légitime de discuter ainsi, entre hommes et entre inconnus, des mérites des grandes dames, surtout de dévaloriser la reine Guenièvre en son propre royaume de Logres ? Peut-on même parler de quelque chose sans être prêt à se battre pour défendre son opinion?

Les dialogues-scènes se terminent enfin comme les autres actions par l'abandon du /vouloir parler/ des personnages et par leur désengagement : le récit de Guillaume se termine par un infléchissement des propos tenus. Ils prennent un tour conclusif, apportant la réponse à la question posée au début, que nous reproduisons ici :

\begin{tabular}{|l|l|}
\hline engagement & désengagement \\
\hline Et se le prie [li cuens] doucement & « Pour ce, fait-il, biaus gentils sire, \\
K'il li die la verité & Ai jou cestui destruit par fu. » (7660-7661) \\
Dont cist mautalent ot esté & \\
Pour qu'il avoit le cuer mangié & \\
De l'escoufle... (7388-7392) & \\
\hline
\end{tabular}

63 Le temps utilisé change, Guillaume abandonne le passé simple de son récit au profit du passé composé, qui signale la reprise en charge de 
l'énonciation: Guillaume-narrateur reprend le pas sur Guillaumepersonnage, mais ce retour du discours sur le récit signale en même temps un désengagement de l'action et fait basculer le récit dans son achèvement. De plus ces propos prennent un tour évaluatif, appelant un jugement (sur le contenu du récit et non sur la manière de le mener) :

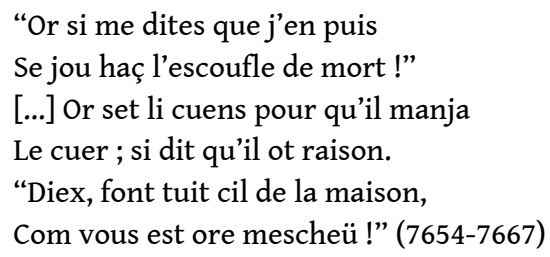

Le processus bascule donc dans l'achèvement par le désengagement du conteur qui signale qu'il a fini de raconter et demande une évaluation. S. Durrer avait expliqué que le dialogue didactique s'arrêtait quand « les interlocuteurs parviennent à une position [discursive] commune, l'inégalité initiale est comblée à la fin de l'échange ${ }^{35}$. » Le narrateur souligne ici nettement que le transfert d'information par le dialogue est terminé. Dans le Merlin, la fin de ce transfert se manifeste par le passage d'un acte directeur représentatif à un acte directif :

\footnotetext{
"Et se vos me voulez croire, nos establirons la tierce ou non de la Trinité, car la Trinitez senefie touz jorz par trois. Et je vos creant, se vos le faites, que granz biens et granz honors vos en vendra a l'ame et au cors et si avendront a vostre tens tels choses dont vos vos merveilleroiz molt. Et se vous la voulez faire, je vos en aiderai et se vos creant que, se vos le faites, que ce sera chose dont il sera molt parlé au pueple, quar molt a doné Nostre Sire grant sens a toz cels qui bien en savront parler. [...] Et se vos me creez, vos en feroiz contre ces choses ce que je vos lo, et se vos le faites et vos me voulez croire, vos en seroiz encore molt loez."

Einsi ot Uitierpandragon oï parler Merlin, si li plost et abelist et si respont :

"Je ne voil pas que Nostre Sire perde noiant por moi de chose qui a sa volanté doie estre faite, ainz voil bien que il saiche que je le met dou tout sur toi ne tu ne me comenderas ja chose que je ne la face, se faire la puis."

Einsis remist li rois seur Merlin qui molt en fu liez. Et Merlins dit :

"Sire, or esgardez ou ele vous plera plus a faire."

Et Uiters respont :

"Je voil qu'ele soit faite la ou tu plus l'ameras..." (Merlin, 48, 75-92; 49, 1-10)
}

Le désengagement de la conversation se fait progressivement par l'engagement de plus en plus marqué dans un nouveau faire, pragmatique cette fois. Le passage des temps du récit à ceux du futur, l'insistance de la formulation hypothétique illustrent ce nouveau départ. L'achèvement de cette scène est catalyseur de l'action suivante. Dans ces deux exemples, le désengagement se produit dans le dialogue par une décision explicite de passer à autre chose et par le dialogue grâce à l'achèvement du processus de transfert d'informations. Dans l'exemple du Tristan, l'achèvement de la conversation n'est pas directement raconté, seul le désengagement de Lancelot est rapporté. Attristé par la nouvelle de la folie de Tristan, il replonge dans le mutisme qu'il avait quitté, sans signaler qu'il se désengage de l'action :

Quant Lanselos entent ceste nouvele, il est tant durement iriés k'il n'a nul pooir de respondre ne onques puis ne lour dist un mot de toute cele nuit, ains pensa toute nuit a cele grant mesqueanche ki ensi estoit avenue a cel boin cevalier. (id., 110, 30-33) 


\section{est simplement résumée :}

Entour la mie nuit, quant li cevalier orent assés parlé d'unes coses et d'autres, il

s'endormirent dusques a l'endemain que li jours aparut biaus et clers. (id., 111, 1- 3)

Les séquences phatiques jouent particulièrement ce rôle d'engagement et de désengagement d'une action et donc d'un dialogue considéré comme action :

Une jeune fille, envoyée par Yseut à la recherche de Tristan s'informe des dernières nouvelles auprès de Palamède.

Quant ils ont grant piece parlé ensamble du roi March et de la roïne Yseut, la damoisele dist a Palamidés :

"Sire cevaliers, j'ai chi tenu une grant piece parlement a vous, tant que assés i ai demouré. Je me voeil anuit mais de vous partir et aler ma voie, car mout ai aillours a faire.

- Madamoisele, fait Palamidés, se vous anuit mais vausissiés demourer pres de moi, or saciés que je vous feïsse herbergier bien et richement et assés pres de chi ; trop en fuisse liés, se Diex me doinst boine aventure, se vous vausissiés remanoir en tel maniere que je vous peüsse cestui soir hounerer et servir.

- Sire, fait la damoisele, mout de mercis de la volenté que vous en avés. Je vous di loiaument que je remansisse trop volentiers se je peüsse, mais je ne puis, vraiement le saciés vous."

Lors vient a son palefroi et monte sans nul autre delaiement faire et conmande a Dieu Palamidés, ne ne veut veut onques k'il le convoit en sus de la fontainne ne poi ne grant. (Tristan 2, 85, 46-63)

La demoiselle manifeste directement sa volonté de mettre fin à cette conversation. La tentative de Palamède de la faire revenir sur sa décision échoue. Mais nous savons que les séquences phatiques complètes sont rares et en particulier à la fin d'un dialogue, on ne s'étonnera pas de la moindre importance donnée à ce moment du désengagement.

Le dialogue-scène est donc un cas particulier de dialogue qui prend l'importance d'une action structurée comme telle par son développement exceptionnel. L'impact que lui donne cette structuration fait de lui en général l'un des moments clés du récit. C'est pourquoi, ces dialogues, à l'échelle de l'intrigue sont plutôt des catalyseurs de l'action, des noyaux pour reprendre la terminologie de R. Barthes. Seul le Tristan donne ce développement à des dialogues analyseurs qui n'ont que peu d'influence sur l'enchaînement des actions, manifestant le pur plaisir des personnages (et du narrateur) à parler sans cesse. dramatique dans le tableau suivant. 


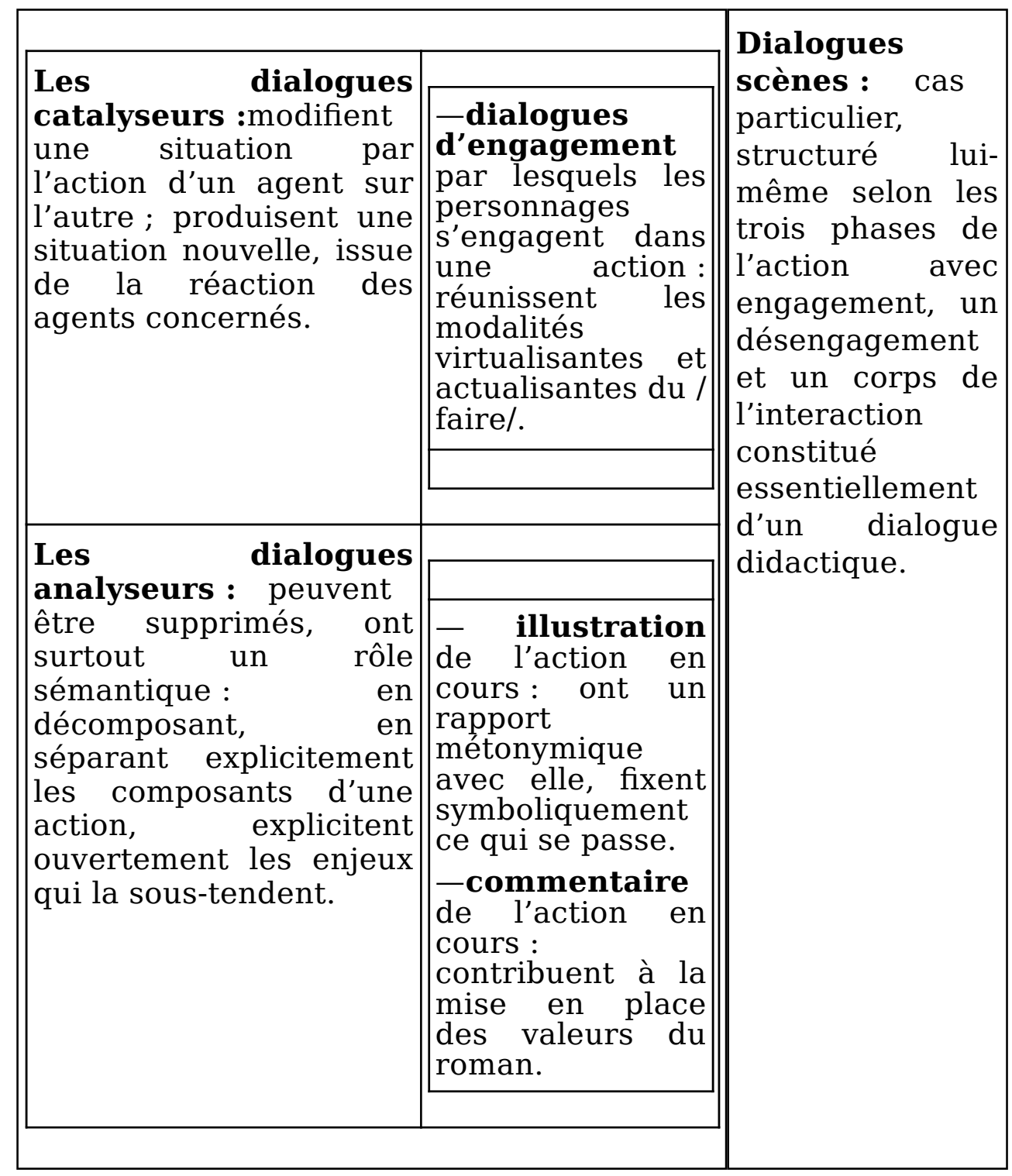

Tableau récapitulatif des différents types de dialogues

Comparaison avec les dialogues des romans modernes

En 1976, J.C. Payen appelait de ses vœux une comparaison du rôle dramatique des dialogues dans les romans anciens et les romans modernes :

Il serait très utile d'entreprendre une étude comparative, qui confronterait les œuvres du Moyen Âge avec le roman moderne où les passages locutifs semblent à première vue moins nombreux, et où ils ont une fonction moins "opératoire ". Le discours fait-il souvent avancer l'action chez un Balzac ou chez un Flaubert ? On a l'impression que le recours aux séquences parlées constitue chez eux non un moteur narratif mais plutôt un simple point de vue sur l'événement: celui du personnage qui ressent le choc de cet événement et réagit par une réponse plus ou moins affective dont la formulation au style direct ou indirect exerce un effet nul ou quasi nul sur la suite de l'intrigue. La littérature narrative du Moyen Âge s'élabore sur une chaîne locutive primordiale parce que c'est une littérature où la 
communication est peut-être plus importante que l'action. L'action ne rebondit souvent que grâce à la rupture ou au rétablissement de cette communication, et plus que l'événement lui-même, compte la façon dont il est perçu et surtout dit par le personnage ${ }^{36}$.

72 La différence se trouve peut-être là : il nous semble, à une lecture que d'aucuns pourront juger encore trop superficielle et simplificatrice, que les grands romans de la modernité, qui ont formé notre jugement, utilisent les paroles de personnages proportionnellement plus comme analyseur que comme catalyseur. Sylvie Durrer ${ }^{37}$ note rapidement à ce sujet une opposition entre un Balzac et un Flaubert ou un Zola. Le premier maintient pour l'essentiel le rôle des dialogues comme éléments de l'action, alors que pour les deux autres, la parole accompagne l'action essentiellement dans une fonction idéologique en renforçant la description d'un personnage, d'une ambiance... que l'on pense par exemple au fameux «Charbovarri » du début du roman de Flaubert, aux interminables discours d'Homais jusqu'au chevet du lit de mort d'Emma. On pourrait dire la même chose des bavardages mondains des soirées proustiennes... Non pas que les auteurs modernes aient négligé le rôle du dialogue dans la progression de l'action, mais ils ont donné une autre importance à des conversations qui, pour les personnages, peuvent paraître insignifiantes mais qui sont nettement révélatrices d'une situation. Dans l'Assommoir, la demande en mariage de Coupeau à Gervaise est peu développée en comparaison avec la scène qui suit immédiatement dans laquelle le jeune couple rencontre les Lorilleux, scène qui préfigure déjà l'hostilité larvée à laquelle ils seront en butte dans le quartier ${ }^{38}$. De même, la décision de Gervaise de vendre sa boutique disparaît au milieu de la longue scène où les personnages veillent le corps de Mme Coupeau en se racontant des histoires ou des fadaise $^{39}$. Il semblerait que les dialogues cruciaux disparaissent au milieu de tous ceux qui ont pour fonction essentielle, non d'engager les personnages les uns par rapport aux autres, mais de dépeindre des rapports humains à la fois insignifiants et sur-signifiants. En revanche, les romans médiévaux présentent essentiellement le dialogue comme une action insérée au milieu des autres avant d'être le symbole d'une situation. Les textes modernes mettent en place un autre rapport entre le récit et les paroles de personnages: elles ne constituent plus un événement, une rupture dans la trame du récit, elles sont déduites de ce qui a été dit par le narrateur. Comme elles ont davantage une fonction d'illustration, elles sont davantage dans la logique de ce qui précède. Par exemple, la description de l'appartement dans lequel s'installent avec bonheur Gervaise et Coupeau au début de leur vie de couple est immédiatement suivie d'un commentaire qui illustre l'admiration des personnages pour leur nouveau domicile :

Le ménage vécut dans l'enchantement de sa nouvelle demeure. [...] La cuisine était grande comme la main et toute noire ; mais en laissant la porte ouverte, on y voyait assez clair; puis, Gervaise n'avait pas à faire des repas de trente personnes, il suffisait qu'elle y trouvât la place de son pot-au-feu. Quant à la grande chambre, elle était leur orgueil. [...] Ensuite, Coupeau avait orné les murs de son mieux, en se promettant des embellissements : une haute gravure représentant un maréchal de 
France [...]; au dessus de la commode, les photographies de la famille étaient rangées sur deux lignes [...] C'était vraiment une belle chambre.

«Devinez combien nous payons ici? » demandait Gervaise à chaque visiteur.

Et quand on estimait son loyer trop haut, elle triomphait, elle criait, ravie d'être si bien pour si peu d'argent:

«Cent cinquante francs, pas un liard de plus !... Hein! C'est donné !»

La rue Neuve de la Goutte d'Or elle-même entrait pour une bonne part dans leur contentement... (l'Assommoir, p. 127)

Léchange de Gervaise et de ses visiteurs (indéfinis) est préparé par les adjectifs et les compliments valorisants qui annoncent le jugement qu'elle porte. Il est vrai que chez Zola, le discours direct se déroule dans le droit fil de la parole de personnage qui envahit toute l'énonciation par le discours indirect libre. De plus, dans ce cas précis, ce dialogue est itératif et reste peu développé par la mise en retrait des divers interlocuteurs de Gervaise. Il renvoie à ce que Danielle Coltier appelle la fonction d'attestation du dialogue, qui, de notre point de vue, correspond à un dialogue analyseur profondément inséré dans le récit. Le phénomène est encore plus net chez Flaubert :

Léon vient retrouver Emma Bovary dans la chambre d'une auberge, il lui avoue son amour.

Et il cherchait comment renouer le dialogue interrompu quand elle lui dit :

«D'où vient que personne, jusqu'à présent, ne m'a jamais exprimé des sentiments pareils?»

Le clerc se récria que les natures idéales étaient difficiles à comprendre. Lui, du premier coup d'œil, il l'avait aimée; et il se désespérait en pensant au bonheur qu'ils auraient eu si, par une grâce du hasard, se rencontrant plus tôt, ils se fussent attachés l'un à l'autre d'une manière indissoluble.

«J'y ai songé quelquefois, reprit-elle.

- Quel rêve ! » murmura Léon.

Et, maniant délicatement le liseré bleu de sa longue ceinture blanche, il ajouta :

"Qui nous empêche donc de recommencer?...

- Non, mon ami, répondit-elle. Je suis trop vieille... vous êtes trop jeune... oubliez moi ! D'autres vous aimeront... vous les aimerez.

- Pas comme vous! s'écria-t-il.

- Enfant que vous êtes! Allons, soyons sage ! Je le veux!»

Et elle lui représenta les impossibilités de leur amour, et qu'ils devaient se tenir, comme autrefois, dans les simples termes d'une amitié fraternelle.

Était-ce sérieusement qu'elle parlait ainsi? Sans doute qu'Emma n'en savait rien elle-même, tout occupée par le charme de la séduction et la nécessité de s'en défendre ; et, contemplant le jeune homme d'un regard attendri, elle repoussait doucement les timides caresses que ses mains frémissantes essayaient. [...] Alors se penchant vers la pendule comme pour regarder l'heure :

"Qu'il est tard, mon Dieu! dit elle; que nous bavardons!»

Il comprit l'allusion et chercha son chapeau.

«J'en ai même oublié le spectacle! Ce pauvre Bovary qui m'avait laissée tout exprès! M. Lormeaux, de la rue Grand-pont, devait m'y conduire avec sa femme. »

Et l'occasion était perdue, car elle partait dès le lendemain.

«Vrai ? fit Léon.

- Oui.

- Il faut pourtant que je vous voie encore, reprit-il, j'avais à vous dire...

- Quoi?

- Une chose... grave, sérieuse. Eh! non, d'ailleurs, vous ne partirez pas, c'est impossible! Si vous saviez... Écoutez-moi !... Vous ne m'avez donc pas compris? Vous n'avez donc pas deviné ?... 
- Cependant vous parlez bien, dit Emma.

- Ah! des plaisanteries! Assez, assez! Faites, par pitié que je vous revoie... une

fois... une seule.

- Eh bien !...»

Elle s'arrêta, puis, comme se ravisant:

«Oh! pas ici!

- Où vous voudrez.

- Voulez-vous...»

Elle parut réfléchir, et d'un ton bref :

« Demain, à onze heures, dans la cathédrale.

- J'y serai ! » s'écria-t-il en saisissant ses mains qu'elle dégagea.

Et, comme ils se trouvaient debout tous les deux, lui placé derrière elle et Emma baissant la tête, il se pencha vers son cou et la baisa longuement à la nuque.

«Mais vous êtes fou! Ah! vous êtes fou! disait-elle avec de petits rires sonores, tandis que les baisers se multipliaient.

Alors, avançant la tête par dessus son épaule, il sembla chercher le consentement de ses yeux. Ils tombèrent sur lui, pleins d'une majesté glaciale.

Léon fit trois pas en arrière pour sortir. Il resta sur le seuil. Puis il chuchota d'une voix tremblante :

« À demain. »

Elle répondit par un signe de tête, et disparut comme un oiseau dans la pièce à côté.

(Madame Bovary ${ }^{40}$, pp. 272-274)

74 Cette scène est nettement singulative, elle débouche sur un changement net de la progression dramatique. Elle engage les personnages dans un rendez-vous qui sera la première étape de l'adultère, en ce sens le dialogue sert de catalyseur à l'action à venir et, par ailleurs, par l'importance accordée à la révélation de l'amour de Léon pour Emma, on pourrait aussi la qualifier de dialogue-scène, en dehors duquel rien n'existerait, mais ce dialogue n'existe même pas aux yeux de Flaubert : il ne vaut pas en soi pour une action ou pour un événement. Cette déclaration d'amour est disqualifiée, soumise à l'ironie sévère de l'auteur : elle n'est pas un événement, un acte libre que commettraient deux individus responsables de ce qu'ils se disent. Le langage que parlent les personnages est aliéné et renvoie aux lieux communs et aux banalités d'une passion naïvement simulée. Le dialogue a moins pour but de parvenir à un changement de situation, ou à représenter ce changement, qu'à illustrer les faux-semblants des comédies humaines, « la platitude et la vanité de toute communication ${ }^{41} »$. Le rapport du dialogue au récit semble donc différent dans ces romans modernes. Il est moins souvent une action s'inscrivant au sein des autres actions du récit qu'une image emblématique des rapports humains et, à ce titre, il illustre surtout la vision de l'homme parlant que le roman veut présenter. Sa fonction idéologique prime en ce qu'il reflète l'univers mental mis en place par l'auteur.

Dans la littérature médiévale au contraire, c'est la force performative de la parole qui est mise en avant et c'est sa fonction dramatique qui domine. À partir d'elle, on retrouve les autres fonctions narratives des dialogues qui lui sont subordonnées. Assurant la structuration de l'histoire en actions qu'elle permet de faire passer à l'acte, elle a une grande importance au niveau de la cohésion du récit. De plus, 
l'engagement adoptant des modalités différentes selon les différents acteurs de l'intrigue et selon leur rapport de force, le dialogue se fait aussi descripteur des personnages. Par ailleurs, les commentaires portés par les personnages sur l'action permettent de guider l'interprétation du narrataire et constituent une part non négligeable de la construction idéologique de l'œuvre. Et cette fonction idéologique des dialogues se renforce enfin d'une cinquième fonction, émotive cette fois, qui manifeste, par les affects qu'ils suscitent chez le narrataire, les normes morales et sociales de l'univers décrit. Ainsi, il nous semble que nous pouvons hiérarchiser les fonctions du dialogue dans les récits médiévaux, à partir de leur fonction dramatique, qu'elles complètent et dont elles dépendent.

$\mathrm{Au}$ Moyen Âge, la fonction dramatique du dialogue est certainement la première en importance dans les récits : les personnages y verbalisent leurs engagement et sont parfaitement conscients de la responsabilité que leur donnent leurs actes de langage au milieu de leurs autres actes. L'homme parlant n'y est pas dépossédé de sa parole par le poids de la société, il agit avec et sur les autres au moyen d'une parole qui n'est pas toujours innocente, pas toujours dépourvue de tout soupçon, mais qui n'est jamais anodine ni insignifiante. Cette force n'est pas seulement une manière élégante et économique d'engager une action dramatique, elle renvoie, comme l'explique Danièle James-Raoul ${ }^{42}$, à une conception plus générale de la parole comme engagement dans le siècle: "S'en cest siecle veus vivre en pais, oï et escoute et si te tais ${ }^{43}$. 》 dit un proverbe. En ce sens, choisir d'intégrer des dialogues à une action renvoie à une certaine conception de la liberté et de la volonté humaine, c'est ce qui donne aux dialogues leur fonction fortement axiologique et idéologique.

\section{NOTES}

1. Maurice Blanchot, Le Livre à venir, Gallimard, Paris, 1959, collection Folio essais, p. 208-209.

2. Danielle COLTIER, "Introduction aux paroles de personnage : fonctions et fonctionnement ", Pratiques ${ }^{\circ} 64$, décembre 1989, p. 69-109.

3. John L. AUSTIN, How to Do Things with Words, 1955, traduction de Gilles LANE, Quand dire, c'est faire , édition française, Paris, Seuil, 1983.

4. Chrétien de troyes, Le Conte du Graal, (vers 1190) éd. Charles mélA, Paris, Le Livre de Poche, 1990.

5. Le Lancelot en prose (1220-30) texte présenté par François Mosès, d'après l'édition d'Elspeth KENNEDY, Paris, Le Livre de Poche, 1991.

6. Robert de BORON, Merlin en prose (env. 1200), éd. Alexandre MichA, Genève, Droz, 1979.

7. Jean-Michel ADAM, L'Analyse des récits, Paris, Seuil, 1996, p. 14.

8. Claude BRÉMOND, Logique du récit, Paris, Seuil, 1973, p. 131.

9. Le Roman de Tristan en prose, tome 1, éd. Philippe MÉNARD, Genève, Droz, 1987. 
10. On notera que Kahedin s'engage déjà à combattre dans les pensées, rapportées, à l'instar d'une parole, au discours indirect et au discours indirect libre. Par ailleurs, le défi ici est peutêtre plutôt un avertissement car le rôle de gardien de pont, que joue Hoël, lui déplait fortement.

11. ChrÉTIEN DE TROYES, Yvain ou le Chevalier au lion, (autour de 1176) éd. David F. HULt, Paris, Le Livre de poche, 1994.

12. Paul RICEUR, Temps et Récit 1, Paris, Seuil, 1983, p. 130.

13. Denis BERTRAND, Précis de sémiotique littéraire, Paris, Nathan, 2000, p. 174.

14. Le Tristan en prose, tome 2, éd. Marie-Luce CHÊNERIE et Thierry DELCOURT, Genève, Droz, 1990.

15. Gautier d'Arras, Éracle, éd. Guy RAYNAUD DE LAGE, Paris, Champion, 1976.

16. Au sens de A. J. GreimAS, "Les Acquis et les projets ", Préface, 1976, in Joseph courtès, Sémiotique narrative et discursive, Paris, Hachette, éd. 1993, p. 5-25, p. 22. Voir plus bas.

17. La Fille du comte de Pontieu, éd. C. BRUNEL, Paris, Champion, 1926

18. Paul zumthor, La Lettre et la voix: de la littérature médiévale, Paris, Seuil, 1987, p. 83.

19. Mauricio веUснот, «Le carré de Saint Anselme et le carré de Greimas ", in Éric LANDowsKI, Lire Greimas, Limoges, Presses Universitaires de Limoges, 1997, p. 15-28, p. 23.

20. Jean-Michel ADAM, op.cit., p. 61.

21. Roland BARTHES, "Introduction à l'analyse structurale des récits ", Communications 8, 1966, p. 828-865.

22. A. J. GReimAS, "Les Acquis et les projets", Préface, 1976, in Joseph Courtès, Sémiotique narrative et discursive, Paris, Hachette, éd. 1993., p. 5-25, p. 23.

23. «alors que le sujet engage dans la transaction la totalité de son faire et de son être, le Destinateur, souverain généreux, s'il offre tout, n'y perd rien de sa substance. »

24. Op.cit. paragraphe 5, p. 8-9.

25. ROBERT DE BORON, Merlin en prose, éd. Alexandre MICHA, Genève, Droz, 1979.

26. Denis BERTRAND, op. cit., p. 189.

27. A. J. GREIMAS, Maupassant, La sémiotique du texte : exercices pratiques, Paris, Seuil, 1976.

28. Le Lancelot en prose, texte présenté par François Mosès, d'après l'édition d'Elspeth Kennedy, Paris, Le Livre de Poche, 1991.

29. Percy LUввоск, The Craft of Fiction, London, Jonathan Cape, 1921, « The art of fiction does not begin until the novelist thinks of his story as a matter to be shown to be so exhibited that it will tell itself. », p. 62.

30. Roland Barthes, art. cit., p. 841.

31. Jean RENART, l'Escoufle (1200-1205), éd. Franklin SWEETSER, Genève, Droz, 1974.

32. Denis Bertrand, op.cit., p. 180.

33. A. J. GREIMAS, «Les Acquis et les projets ", Préface, 1976, in Joseph courTÈs, Sémiotique narrative et discursive, Paris, Hachette, éd. 1993., p. 5-25, p. 24.

34. Passage qui s'étend du vers 7360 au vers 7700 .

35. Sylvie DURRER, Le Dialogue romanesque, Genève, Droz, 1994, p. 138.

36. Jean-Charles PAYEN, «Le clos et l'ouvert dans la littérature française, médiévale et les problèmes de la communication ", Perspectives médiévales 2, Paris, 1976, p. 61-72, p. 68.

37. S. DURRER, op. cit., p. 249.

38. Émile zoLA, L'Assommoir, Folio Gallimard, 1978, p..

39. id. p.

40. Flaubert, Madame Bovary, Le Livre de Poche, 1983.

41. S. DURRER, op. cit., p. 249.

42. Danièle JAMES-RAOUL, La Parole empêchée, Paris, Champion, 1997, p. 112.

43. MORAWSKI 2254, cité par D. James-Raoul, id., p. 121. 


\section{RÉSUMÉS}

Les romans médiévaux se caractérisent par un rôle structurel très fort des dialogues. On peut classer ceux-ci en deux catégories selon leur fonction dans l'action, et quel que soit le type de discours dont ils étaient constitués. Première catégorie : les dialogues catalyseurs. Ils modifient une situation, soit en engageant les personnages dans l'action par un ordre, une décision... soit en les désengageant de l'action quand ils l'estiment terminée. Ils produisent un composé nouveau avec les données de l'action. La deuxième catégorie regroupe les dialogues analyseurs, qui explicitent l'action. Ils peuvent être supprimés au plan des événements mais pas au plan du contenu sémantique de l'œuvre. Soit ils illustrent la scène en ajoutant une parole emblématique, soit ils la commentent. Certains dialogues, particulièrement longs, font figure de scène en euxmêmes et comportent les mêmes phases d'engagement et de désengagement que les actions d'une autre nature. C'est une troisième catégorie qui recoupe les deux autres. Cette typologie est indépendante de la qualité présumée des dialogues.

Medieval romances are characterized by a very important structural function of the dialogues. They can be classified into two categories according to their function in the action and regardless of the reported speech they use. The first category is the catalyst dialogues, which modify a situation either by engaging the characters in the action through an order or a decision, or by freeing them from the action, when it is considered as having ended. These dialogues create a new combination by merging components of the action. The second category is composed of analytic dialogues, which clarify the action. They can be removed from the story with respect to the action but not meaning. They either illustrate the scene by adding emblematic language, or they comment on it. Some particularly long dialogues act as a scene in themselves and are made up with the same phases of involvement and disengagement. This third category intersects the two others. This typology is not linked to the size or the literary merit of the dialogues.

\section{INDEX}

Index géographique : France

Mots-clés : dialogues, fonctions narratives, romans médiévaux

Keywords : dialogues, medieval romances, narrative functions

Index chronologique : Moyen Âge

\section{AUTEUR}

\section{CORINNE DENOYELLE}

Université de Toronto 